\title{
Detecting resonant tidal excitations of Rossby modes in coalescing neutron-star binaries with third-generation gravitational-wave detectors
}

\author{
Sizheng Ma $\odot,{ }^{*}$ Hang Yu $\odot{ }^{\dagger}$ and Yanbei Chen ${ }^{\ddagger}$ \\ TAPIR, Walter Burke Institute for Theoretical Physics, California Institute of Technology, \\ Pasadena, California 91125, USA
}

(Received 6 October 2020; accepted 12 February 2021; published 15 March 2021)

\begin{abstract}
Rossby modes ( $r$-modes) of rotating neutron stars can be excited by the gravitomagnetic forces in coalescing binary systems. A previous study by Flanagan and Racine [Phys. Rev. D 75, 044001 (2007)] showed that this kind of dynamical tide (DT) can induce phase shifts of $\sim 0.1 \mathrm{rad}$ on gravitational waveforms, which is detectable by third-generation $(3 \mathrm{G})$ detectors. We study the impact of this DT on measuring neutron-star parameters in the era of $3 \mathrm{G}$ detectors. We incorporate two universal relations among neutron-star properties predicted by different equations of state: (i) the well-known I-Love relation between momentum of inertia and ( $f$-mode) tidal Love number, and (ii) a relation between the $r$-mode overlap and tidal Love number, which we newly explore. We find that $r$-mode DT will provide rich information about slowly rotating neutron stars with frequency $10-100 \mathrm{~Hz}$ and spin inclination angle $18^{\circ}-110^{\circ}$. For a binary neutron-star system (with a signal-to-noise ratio 1500 in the Cosmic Explorer), the spin frequency of each individual neutron star can be constrained to 6\% (fractional error) in the best-case scenario. The degeneracy between the Love numbers of individual neutron stars is dramatically reduced: each individual Love number can be constrained to around $20 \%$ in the best case, while the fractional error for both symmetric and antisymmetric Love numbers are reduced by factors of around 300. Furthermore, DT also allows us to measure the spin inclination angles of the neutron stars, to $0.09 \mathrm{rad}$ in the best case, and thus place constraints on neutron-star natal kicks and supernova explosion models. In addition to parameter estimation, we also develop a semianalytic method that accurately describes detailed features of the binary evolution that arise due to the DT.
\end{abstract}

DOI: 10.1103/PhysRevD.103.063020

\section{INTRODUCTION}

Gravitational-wave (GW) astronomy recently provided us a new way to study neutron-star (NS) physics, with events GW170817 [1,2] and GW190425 [3] already imposing new constraints on NS properties [4-12]. With future upgrades for Advanced LIGO and Virgo [13-24], LIGO-India [25], and KAGRA [26,27], as well as thirdgeneration detectors like the Einstein Telescope [28-31] and the Cosmic Explorer (CE) [32,33], we expect to detect more events, as well as to achieve much higher signal-tonoise ratios [34]. These new opportunities supply motivation for the more accurate modeling of NSs in coalescing binaries, and the GWs they emit [35-40].

During the last few minutes of a binary neutron-star inspiral, the orbital frequency sweeps through tens of hertz to hundreds of hertz; internal fluid motions of NSs may get resonantly excited by tidal forces exerted by their companions. Such fluid motion will also act back onto the orbital motion. This phenomenon is called the dynamical

\footnotetext{
ssma@caltech.edu

hangyu@caltech.edu

¥yanbei@caltech.edu
}

tide (DT), and it was first investigated by Cowling [41]. For nonspinning NSs in binaries with circular orbits, only $g$-modes can be resonantly excited, but their effect on gravitational waveforms is negligible [42-45]. However, DT can be enhanced by orbital eccentricity [46-49] and rotation [50-53].

The effect of rotation on DTs was first studied by Lai [50,51], who aimed to explain the orbital decay of the PSR J0045-7319/B binary system. Lai pointed out that stellar rotation can excite $f$-modes and lower-order $g$-modes. Since these modes couple more strongly to the tidal field, DTs should be more pronounced. The same formalism was then applied to coalescing binaries by Ho and Lai [52] to investigate the impact of DTs on GWs. It was found that $f$-mode resonances require NSs to rotate at very high frequencies-and only in this case does it induce a significant $\mathrm{GW}$ phase shift. On the other hand, $g$-mode resonances are still too weak to be detected. Several authors later extended the above Newtonians studies and discussed the excitation of $f$-modes and $g$-modes in the context of general relativity [35,54-58].

Although $f$-mode resonances can significantly influence GWs, the high rotation rate required here is disfavored by 
the astrophysics of formation scenarios. It is generally believed that NSs in binaries have already spun down to low frequencies $(\lesssim 40 \mathrm{~Hz})$ when their $\mathrm{GW}$ frequencies enter the band of ground-based detectors [59,60]. For example, recent events GW170817 [1] and GW190425 [3] were both consistent with low spins. In this way, $f$-mode resonance might not be very promising for $\mathrm{GW}$ observations.

For spinning neutron stars, in addition to $f-, p$-, and $g$-modes, there are also inertial modes (hybrid modes) that are mainly supported by the Coriolis force [61-63]. As pointed out by Lockitch and Friedman [62], for isentropic stars, ${ }^{1}$ rotation mixes purely axial modes and purely polar modes and leads to a class of modes which have hybrid parity, where each of them can be classified into axial-led or polar-led modes. For Newtonian stars, there is a special subclass of modes that has a purely axial parity which is usually referred to as the Rossby modes (or $r$-modes) $[64,65]$, although this subclass also obtains a polar part for relativistic stars [66-68]. On the other hand, for nonisentropic NSs, the mixing of $g$-modes and $r$-modes takes place at a relatively high rotation rate (where the Coriolis force dominates over the buoyancy), leading to the socalled inertial-gravity modes.

Previous studies have shown that inertial modes (including $r$-modes) and inertial-gravity modes can be excited by the (gravitoelectric) Newtonian tidal field $[52,69,70]$. The resonance takes place as the orbital angular velocity becomes comparable to the spin frequency, which requires a minimum NS spin of only tens of hertz. Although this spin requirement is more than likely fulfilled, the effect of such resonances is too weak to be detectable. However, inertial modes (including $r$-modes) can also be excited by the gravitomagnetic force [71-75]. As pointed out by Flanagan and Racine (hereafter FR07) [72], this kind of DT can induce $\sim 0.1 \mathrm{rad}$ of GW phase shift-detectable by third-generation detectors like the Einstein Telescope and CE. Their studies, though, did not provide detailed discussions on how those DTs impact parameter estimation. Later on, Yu et al. [76] proposed that the $r$-mode DTs could improve the accuracy of tidal Love number measurements. The aim of this paper is to build upon FR07 and Ref. [76] and study the $r$-mode DTs in more detail, including a Fisher-matrix analysis. Specifically, we will investigate the impact of universal relations among NS properties [77,78].

This paper is organized as follows. We first give a summary on the implication of $r$-mode DTs on GW parameter estimation in Sec. II, and the rest of the paper presents the details. In Sec. III, we briefly review the coupling between the $r$-mode and the gravitomagnetic

\footnotetext{
${ }^{1}$ Isentropic stars have no buoyancy; both $g$-modes and $r$-modes (purely axial modes) have a vanishing mode frequency in the nonspinning limit, and they are mixed even without rotation.
}

force. In Sec. IV, we discuss features of $r$-mode excitation and the $r$-mode's impact on the orbital evolution. We first focus on the orbital part in Sec. IV B, where we compare the model presented in FR07 [Eq. (1)] with numerical integration. Next we study the tidal excitation in Sec. IV C, where we give an analytic formula for $r$-mode tidal evolution that is valid in the entire evolution regime. In Sec. V, we explore the universal relation between the normalized $r$-mode overlap and the normalized tidal Love number. Section VI focuses on the construction of gravitational waveforms. We first propose a hybrid GW waveform in Sec. VIA, which incorporates both $r$-mode and other post-Newtonian (PN) effects. Next in Sec. VI B we provide a model for the GW phase of the $r$-mode, following FR07. In Sec. VII, we use the Fisher information matrix formalism to discuss the influence of $r$-mode excitation on parameter estimation. Finally, in Sec. VIII we summarize our results.

Throughout this paper, we use geometric units with $G=c=1$.

\section{SUMMARY OF PARAMETER DEPENDENCIES}

In this section, we shall give a brief summary of $r$-mode resonance's impact on the gravitational waveform, and on how physical parameters of neutron-star and neutron-starblack-hole binaries influence the gravitational waveform via point-particle, spin-orbit, $f$-mode tide, and $r$-mode resonance effects. We shall divide binaries into four categories and discuss in each category how some, or all, of the physical parameters of the neutron stars can be measured.

\section{A. The role of $\boldsymbol{r}$-mode dynamical tide}

As discussed in FR07 [72], $r$-mode excitation is mainly controlled by the spin frequency $\Omega_{s}$ and $r$-mode coupling coefficient $\mathcal{I}$ [Eq. (30)], which play different roles: (i) Spin frequency determines the location (both in time and frequency domains) of resonance during orbital evolution, because resonance takes place as the tidal driving frequency (related to the orbital frequency) coincides with the pattern frequency of the mode (determined by the spin frequency) in the inertial frame. (ii) The $r$-mode coupling coefficient $\mathcal{I}$ characterizes the strength of the $r$-mode DT, therefore determines the effect of this DT on orbital evolution [see Sec. IV for details]. As proposed in FR07, the orbital evolution before and after the $r$-mode resonance can be described as two different point-particle (PP) orbits, with orbitalfrequency evolution given by

$$
\dot{\phi}(t)= \begin{cases}\dot{\phi}_{\mathrm{PP}}^{(\mathrm{pre})}(t), & \text { if } t<t_{r}, \\ \dot{\phi}_{\mathrm{PP}}^{(\mathrm{post})}(t), & \text { if } t>t_{r},\end{cases}
$$


'with $t_{r}$ the time of resonance. ${ }^{2}$ These two frequency evolutions satisfy the same evolution equation, with the same set of parameters (including mass, spin, inclination angles, etc.), which, at Newtonian order, reads

$$
\frac{d}{d t} \dot{\phi}_{(\mathrm{PP})}=\frac{96}{5} \mathcal{M}^{5 / 3} \dot{\phi}_{(\mathrm{PP})}^{11 / 3},
$$

where $\mathcal{M}$ is the chirp mass. The two orbits are related to each other by a "jump" at $t_{r}$

$$
\dot{\phi}_{\mathrm{PP}}^{(\mathrm{post})}\left(t_{r}\right)-\dot{\phi}_{\mathrm{PP}}^{(\mathrm{pre})}\left(t_{r}\right)=\Delta \dot{\phi}_{\mathrm{tide}},
$$

where $\Delta \dot{\phi}_{\text {tide }}$ depends on $\mathcal{I}$ (see Sec. IV B for more details). As a result, by studying the GW emitted from the orbit in Eq. (1), we can obtain the constraints on $\Omega_{s}$ and $\mathcal{I}$.

\section{B. The impact of the $r$-mode DT on parameter estimation}

In order to explore how the additional information from the $r$-mode DT can improve parameter estimation, we consider both binary neutron-star (BNS) and black-holeneutron-star (BHNS) binaries and distinguish whether universal relations between neutron-star properties are used as input. This will divide these binaries into four categories.

\section{BNS systems without an r-mode DT or universal relations}

A spinning BNS system is sketched in Fig. 1. Without an $r$-mode DT (but with an $f$-mode adiabatic tide), the system has six intrinsic parameters: chirp mass $\mathcal{M}$, mass ratio $\Xi=m_{1} /\left(m_{1}+m_{2}\right)$, two individual tidal Love numbers $\lambda_{f}^{(i)}$, and two dimensionless spins along the direction of orbital angular momentum $\chi_{i}^{(z)}$. (The subscript $i=1,2$ labels the two NSs.) In the absence of DT, the system evolves under four main effects: the Newtonian gravity, PN corrections from mass ratio, $\mathrm{PN}$ corrections from spin-orbit (SO) coupling, and the adiabatic ( $f$-mode) tidal effect, as summarized in Table I. Here we do not include the PN spinspin coupling [79] or PN spin precession [80], because their effects are negligible even for third-generation $(3 \mathrm{G})$ detectors for the range of spins that we consider $(10-80 \mathrm{~Hz}$; see Appendix A for more details). For a BNS system, the waveform depends only on a combination of $\lambda_{f}^{(i)}$ and $\chi_{i}^{z}$, not each individually; as a result, we can measure only four parameters to meaningful accuracy: $\mathcal{M}, \Xi, \chi^{\mathrm{eff}}$, and $\lambda_{f}^{\text {eff }}$, with $\lambda_{f}^{\text {eff }}$ and $\chi^{\text {eff }}$ defined by $[81,82]$

\footnotetext{
${ }^{2}$ The resonance condition is given by Eq. (19). Hereafter we use the subscript $r$ to refer to the value at the resonance time.
}

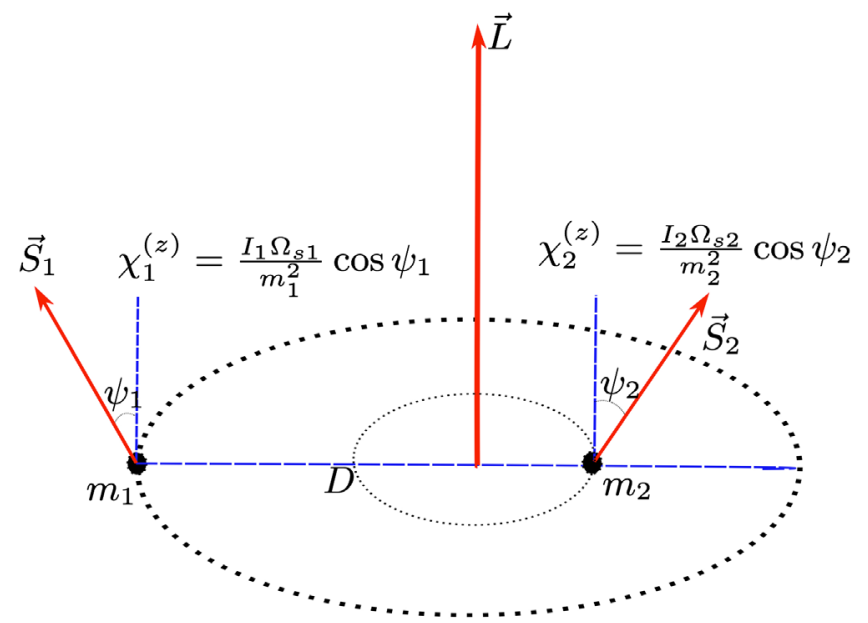

FIG. 1. A BNS system $m_{1}-m_{2}$ with two spin vectors $\vec{S}_{1}$ and $\vec{S}_{2}$. The neutron stars' spin axes are tilted by angles $\psi_{1,2}$ with respect to the direction of the orbital angular momentum $\vec{L}$. Here the azimuthal angle of the spins is unimportant, because the effect of precession is negligible.

$$
\begin{aligned}
& \chi^{\mathrm{eff}}=\frac{m_{1} \chi_{1}^{(z)}+m_{2} \chi_{2}^{(z)}}{M}, \\
& \lambda_{f}^{\mathrm{eff}}=\left(11 \frac{m_{2}}{m_{1}}+\frac{M}{m_{1}}\right) \lambda_{f}^{(1)}+\left(11 \frac{m_{1}}{m_{2}}+\frac{M}{m_{2}}\right) \lambda_{f}^{(2)},
\end{aligned}
$$

where $M=m_{1}+m_{2}$. The two individual Love numbers $\lambda_{f}^{(i)}$, as well as the two $\chi_{i}^{(z)}$, are degenerate. Even among the constrainable parameters, errors in $\Xi, \chi^{\text {eff }}$, and $\lambda_{f}^{\text {eff }}$ are highly correlated with one other. In order to obtain a good constraint on $\lambda_{f}^{\text {eff }}$, a low-spin prior based on the observed galactic binary NS population has to be assumed $[1,4]$. As we shall discuss later, such an assumption is not necessary if $r$-mode resonance can be incorporated.

\section{BNS systems with the I-Love universal relation but without a DT}

For the same BNS system (without a DT), the I-Love universal relation of NSs [77,78] can be used to improve parameter estimation. This is a relation between the momentum of inertia and the tidal Love number that is insensitive to the equation of state (EOS). To use this relation, we express $\chi^{(z)}$ of each NS as

$$
\chi_{i}^{(z)}=\frac{I_{i} \Omega_{s i}}{m_{i}^{2}} \cos \psi_{i}
$$

In this case, we have a total of ten parameters: chirp mass $\mathcal{M}$, mass ratio $\Xi$, two Love numbers $\lambda_{f}^{(i)}$, two moments of inertia $I_{i}$, two inclination angles $\psi_{i}$, and two spin frequencies $\Omega_{s i}$. As listed in Table II ("non-DT sector"), there are six constraints on these parameters: four are from GWs 
TABLE I. Parameters and constraints for a BNS system without an $r$-mode DT and not applying the universal relations between NS properties. We have six parameters and four independent constraints; as a result, the two individual Love numbers are degenerate, and so are the two individual dimensionless spins.

\begin{tabular}{lccc}
\hline \hline All variables in the GW & Effect & Variables in the effect & Constrainable variable \\
\hline $\mathcal{M}, \Xi \lambda_{f}^{(1)}, \lambda_{f}^{(2)} \chi_{1}^{(z)}, \chi_{2}^{(z)}$ & PN (PP part) & $\mathcal{M}$ & $\mathcal{M}$ \\
& PN (PP part) & $\Xi$ & $\Xi$ \\
& SO coupling & $\chi_{1}^{(z)}, \chi_{2}^{(z)}$ & $\chi^{\text {eff }}$ \\
& Adiabatic tide & $\lambda_{f}^{(1)}, \lambda_{f}^{(2)}$ & $\lambda_{f}^{\text {eff }}$ \\
\hline \hline
\end{tabular}

$\left(\mathcal{M}, \Xi, \chi^{\text {eff }}, \lambda_{f}^{\text {eff }}\right)$, as discussed above, and two are from universal relations (each star contributes one constraint). This is still not enough to independently constrain all ten parameters.

Introducing the universal relations did not reduce the number of degeneracies because more parametersnamely, the spin frequency and inclination angle for each NS - are needed to be introduced in order to use these relations. The situation will change as we consider the $r$-mode DT.

\section{Resonant BNS systems with universal relations}

Now take the $r$-mode resonance into account. As shown in Table II, we have in total 12 parameters: $\operatorname{chirp}$ mass $\mathcal{M}$, mass ratio $\Xi$, two Love numbers $\lambda_{f}^{(i)}$, two momenta of inertia $I_{i}$, two spin frequencies $\Omega_{s i}$, two inclination angles $\psi_{i}$, and two $r$-mode coupling coefficients $\mathcal{I}_{i}$. Meanwhile, we can obtain 12 constraints from GWs and the universal relations. Six of them $\left(\mathcal{M}, \Xi, \chi^{\text {eff }}, \lambda_{f}^{\text {eff }}, I_{1,2}\right)$ were already discussed in Sec. II B 2. The rest of the six constraints involve the $r$-mode. Four are constraints on $\Omega_{s i}$ and $\mathcal{I}_{i}$, as we discussed in Sec. II A. The other two constraints come from a new universal relation found in this paper-namely, the relation between the normalized $r$-mode overlap and the normalized Love number (see Sec. V). As a result, the number of constraints are the same as the number of parameters. In principle, this breaks degeneracy and allows us to estimate all parameters independently, given a high enough signal-to-noise ratio. However, as it later turns out, even in the era of $3 \mathrm{G}$ detectors, for each individual binary we still may not fully constrain all parameters with relative error less than $100 \%$, since the DT sector is not strong enough.

\section{Resonant BHNS systems with universal relations}

For BHNS systems, the $r$-mode resonance takes place only once before merger. As shown in Table III, the system has eight parameters: chirp mass $\mathcal{M}$, mass ratio $\Xi$, NS Love number $\lambda_{f}^{(1)}$, NS moment of inertia $I_{1}$, NS spin frequency of NS $\Omega_{s 1}$, NS inclination angle $\psi_{1}$, NS $r$-mode coupling coefficient $\mathcal{I}_{1}$, and the spin of $\mathrm{BH}$ along the direciton of orbital angular momentum $\chi_{2}^{(z)}$. We can obtain eight

TABLE II. Parameters and constraints for a BNS system with universal relations (for both NSs). In the "non-DT sector," when $r$-mode DTs do not take place, we have ten parameters and six constraints, with four degeneracies. In the presence of $r$-mode DTs, we have two more parameters, but six more constraints. As a result, we have in total 12 parameters and 12 constraints; the system can in principle be decoded without degeneracy.

\begin{tabular}{|c|c|c|c|c|}
\hline Variables in GW & Sector & Effect & Variables in effect & Constrainable variable \\
\hline \multirow{5}{*}{$\mathcal{M}, \Xi \lambda_{f}^{(1)}, \lambda_{f}^{(2)} I_{1}, I_{2} \psi_{1}, \psi_{2} \Omega_{s 1}, \Omega_{s 2}$} & \multirow{5}{*}{ Non-DT sector } & PN (PP part) & $\mathcal{M}$ & $\mathcal{M}$ \\
\hline & & PN (PP part) & $\Xi$ & $\Xi$ \\
\hline & & SO coupling & $\begin{array}{c}I_{1}, \Omega_{s 1}, \psi_{1}, \mathcal{M} \\
I_{2}, \Omega_{s 2}, \psi_{2}, \Xi\end{array}$ & $\chi^{\text {eff }}$ \\
\hline & & Adiabatic tide ( $f$-mode) & $\begin{array}{l}\lambda_{f}^{(1)}, \lambda_{f}^{(2)} \\
\lambda_{f}^{(1)}, \lambda_{f}^{(2)}\end{array}$ & $\lambda_{f}^{\mathrm{eff}}$ \\
\hline & & I-Love universal relation & $\begin{array}{c}I_{1}, I_{2} \\
\Xi\end{array}$ & $I_{1}, I_{2}$ \\
\hline \multirow[t]{3}{*}{$\mathcal{I}_{1}, \mathcal{I}_{2}$} & \multirow{3}{*}{ DT sector } & $r$-mode resonances & $\begin{array}{l}\Omega_{s 1}, \Omega_{s 2} \\
\mathcal{I}_{1}, \mathcal{I}_{2}\end{array}$ & $\begin{array}{c}\Omega_{s 1}, \Omega_{s 2} \\
\mathcal{I}_{1}, \mathcal{I}_{2}\end{array}$ \\
\hline & & & $\mathcal{I}_{1}, \psi_{1}, \lambda_{f}^{(1)}$ & \\
\hline & & $r$-mode universal relation & $\begin{array}{c}\mathcal{I}_{2}, \psi_{2}, \lambda_{f}^{(2)} \\
\Xi\end{array}$ & $\psi_{1}, \psi_{2}$ \\
\hline
\end{tabular}


TABLE III. Parameters and constraints for a BHNS system with universal relations and $r$-mode DTs. We have eight parameters and eight constraints, and the system can be decoded without degeneracy.

\begin{tabular}{lccc}
\hline \hline All variables in GW & Effect & Variables in the effect & Constrainable variable \\
\hline $\mathcal{M}, \Xi \lambda_{f}^{(1)}, I_{1} \psi_{1}, \Omega_{s 1} \mathcal{I}_{1}, \chi_{2}^{(z)}$ & PN (PP part) & $\mathcal{M}$ & $\mathcal{M}$ \\
& PN (PP part) & $\Xi$ & $\Xi$ \\
& SO coupling & $I_{1}, \Omega_{s 1}, \psi_{1}$ & $\chi^{\text {eff }}$ \\
& Adiabatic tide $(f$-mode) & $\chi_{2}^{(z)}, \Xi, \mathcal{M}$ & $\lambda_{f}^{(1)}$ \\
& I-Love universal relation & $\lambda_{f}^{(1)}$ & $I_{1}$ \\
& $r$-mode resonances & $\lambda_{f}^{(1)}, I_{1}$ & $\Omega_{s 1}, \mathcal{I}_{1}$ \\
\hline \hline
\end{tabular}

constraints on these parameters, with six of them from GWs $\left(\mathcal{M}, \Xi, \chi^{\text {eff }}, \lambda_{f}^{(1)}, \Omega_{s 1}, \mathcal{I}_{1}\right)$, and two from the two universal relations. In this way, the BHNS system is also expected to be decoded without degeneracy, given a sufficient signalto-noise ratio.

\section{BASIC EQUATIONS OF DYNAMICAL TIDES}

In this section, we briefly review the coupling between $r$-modes and gravitomagnetic force in coalescing binary systems. We refer the reader to the work of Flanagan and Racine [72,83] for further details. In Sec. III A, we first provide some basic information about $r$-modes in NSs. All equations are kept to linear order in spin frequency. In Sec. III B, we show how $r$-modes are driven by the gravitomagnetic force. Finally, in Sec. III C, we discuss the tidal backreaction to the orbit and present the full equation of motion (EOM). In this section, as done in FR07, the slowly rotating NS is treated in Newtonian gravity. This will lead to the correct form of evolution equations, although parameters may eventually need relativistic corrections. We shall incorporate $\mathrm{PN}$ and $f$-mode adiabatic tide corrections using a hybrid approach described in Sec. VI.

\section{A. Rossby modes}

For a rotating NS with mass $m_{1}$, we introduce a corotating frame $\left(x^{\prime}, y^{\prime}, z^{\prime}\right)$, in which the spin of the star $\boldsymbol{\Omega}_{s 1}$ is along the $z^{\prime}$ direction. In this coordinate system, the perturbation equation of the rotating star is given by

$$
\frac{\partial^{2} \boldsymbol{\xi}}{\partial t^{2}}+2 \boldsymbol{\Omega}_{s 1} \times \frac{\partial \boldsymbol{\xi}}{\partial t}+\boldsymbol{C} \cdot \boldsymbol{\xi}=\boldsymbol{a}_{\mathrm{ext}}
$$

where $\boldsymbol{\xi}$ is the Lagrangian displacement of fluid elements, $\boldsymbol{C}$ is a self-adjoint operator, and $\boldsymbol{a}_{\text {ext }}$ is the external driving, which will arise from gravitomagnetism in our case. We refer the interested reader to Ref. [84] for more details.
In the phase-space mode expansion framework [71], $\boldsymbol{\xi}$ and its time derivative $\dot{\xi}$ can be expanded as a summation of modes

$$
\left(\begin{array}{l}
\boldsymbol{\xi} \\
\dot{\boldsymbol{\xi}}
\end{array}\right)=\sum_{l m} c_{l m}(t)\left(\begin{array}{c}
\boldsymbol{\xi}_{l m} \\
-i \omega_{l m} \boldsymbol{\xi}_{l m}
\end{array}\right),
$$

where the angular quantum numbers $l$ and $m$ are integers with $m= \pm l, \pm(l-1) \ldots 0$. Here $\omega_{l m}$ is the corotating frame eigenfrequency of the mode. Each mode $\boldsymbol{\xi}_{l m}$ satisfies the following eigenvalue problem,

$$
-\omega_{l m}^{2} \boldsymbol{\xi}_{l m}-2 i \omega_{l m} \boldsymbol{\Omega}_{s 1} \times \boldsymbol{\xi}_{l m}+\mathbf{C} \cdot \boldsymbol{\xi}_{l m}=0,
$$

and modes with $(l, m) \neq\left(l^{\prime}, m^{\prime}\right)$ satisfy the following orthogonality condition,

$\left\langle\boldsymbol{\xi}_{l m}, 2 i \boldsymbol{\Omega}_{s 1} \times \boldsymbol{\xi}_{l^{\prime} m^{\prime}}\right\rangle+\left(\omega_{l m}+\omega_{l^{\prime} m^{\prime}}\right)\left\langle\boldsymbol{\xi}_{l m}, \boldsymbol{\xi}_{l^{\prime} m^{\prime}}\right\rangle=0$,

with the inner product defined by

$$
\langle\boldsymbol{u}, \boldsymbol{v}\rangle=\int d^{3} x^{\prime} \rho \boldsymbol{u}^{*} \cdot \boldsymbol{v},
$$

where $\rho$ is the density profile of NS.

In presence of driving, the mode amplitudes satisfy

$$
\dot{c}_{l m}(t)+i \omega_{l m} c_{l m}(t)=\frac{i}{b_{l m}}\left\langle\boldsymbol{\xi}_{l m}, \boldsymbol{a}_{\mathrm{ext}}\right\rangle
$$

where the coefficient $b_{l m}$ is given by

$$
b_{l m}=\left\langle\boldsymbol{\xi}_{l m}, 2 \omega_{l m} \boldsymbol{\xi}_{l m}+2 i \boldsymbol{\Omega}_{s 1} \times \boldsymbol{\xi}_{l m}\right\rangle .
$$

To linear order in spin frequency, the $(l, m) r$-mode has a Lagrangian displacement vector field as

$$
\xi_{l m}\left(r^{\prime}, \theta^{\prime}, \phi^{\prime}\right)=-\frac{i f_{l m}\left(r^{\prime}\right)}{\sqrt{l(l+1)}} \boldsymbol{r}^{\prime} \times \nabla Y_{l m}\left(\theta^{\prime}, \phi^{\prime}\right)
$$


where $Y_{l m}\left(\theta^{\prime}, \phi^{\prime}\right)$ are spherical harmonics, and a (corotating frame) eigenfrequency given by [84]

$$
\omega_{l m}=-\frac{2 m \Omega_{s 1}}{l(l+1)} .
$$

The radial profile function $f_{l m}\left(r^{\prime}\right)$ can be determined only if one includes the next-order correction of spin frequency. For barotropic stars, only the modes $|m|=l$ exist, and their coefficients $f_{l m}\left(r^{\prime}\right)$ are given by [84]

$$
f_{l m}\left(r^{\prime}\right) \propto r^{\prime l} .
$$

The constant of proportionality can be determined by the normalization condition

$$
\left\langle\boldsymbol{\xi}_{l m}, \boldsymbol{\xi}_{l m}\right\rangle=m_{1} R_{1}^{2},
$$

where $R_{1}$ is the radius of the star. As for other modes with $|m| \neq l, f_{l m}=0$. Henceforth, we restrict our discussions to barotropic stars and focus on the $(2,2) r$-mode, since it gives the strongest effect [72].

\section{B. Gravitomagnetic coupling: Stellar part}

To investigate the coupling between the $(2,2) r$-mode and the gravitomagnetic force, i.e., the rhs of Eq. (10), we place the spinning NS, $m_{1}$, in a binary system. It is perturbed by the gravitomagnetic tidal field $\mathcal{B}_{i j}(t)$ exerted by the companion $m_{2}$. For convenience, we introduce a nonrotating, comoving coordinate system $(x, y, z)$ centered at $m_{1}$, as shown in Fig. 2, with the $z$ axis placed along the spin axis. The comoving frame is related to the corotating frame (the primed frame) through a rotation about the $z\left(z^{\prime}\right)$ axis by $\Omega_{s 1} t$. (Note that the comoving frame can be nonrotating because we treat the spin directions of NSs as fixed in this paper.)

Using the same convention as Ref. [72], we parametrize the location of $m_{2}, z$ as

$z(t)=D(t)\left[\cos \psi_{1} \cos \phi(t), \sin \phi(t), \sin \psi_{1} \cos \phi(t)\right]$,

where $\psi_{1}$ is the inclination angle between the stellar spin vector and the orbital angular momentum; $D(t)$ is the separation between two NSs. With this parametrization, the orbital angular moment, $\vec{L}$, is in the $x-z$ plane. This plane and the orbital plane intersect at $\vec{N}$. The orbital phase of $m_{2}$, $\phi(t)$ is the angle between $\vec{z}$ and $\vec{N}$.

The expressions of $\mathcal{B}_{i j}(t)$ and $\boldsymbol{a}_{\text {ext }}$ are given in Sec. V C of Ref. [72]. Plugging them and Eq. (12) into Eq. (10), we obtain the evolution equation for $c_{22}$,

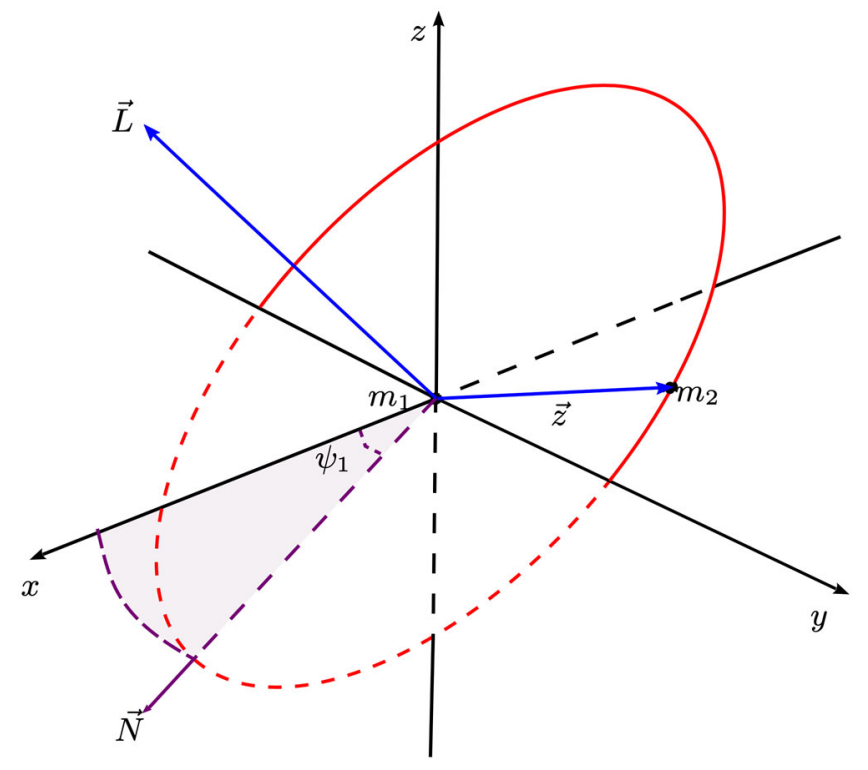

FIG. 2. A comoving coordinate system $(x, y, z)$ that centers at $m_{1}$. The companion NS $m_{2}$ orbits around $m_{1}$, whose orbital plane intersects with the $x-z$ plane at $\vec{N}$ and intersects with the $x-y$ plane at the $y$ axis. The orbital phase $\phi(t)$ is the angle between $\vec{N}$ and the location of $m_{2}, \vec{z}$. The orbital angular momentum $\vec{L}$ is in the $x-z$ plane, with polar angle $\psi_{1}$.

$$
\begin{aligned}
\dot{c}_{22}+i \omega_{22} c_{22}= & \frac{i}{\omega_{22}} \sqrt{\frac{16 \pi}{5}} \frac{I_{22}^{r} m_{2} R_{1}}{D^{2}} \dot{\phi}\left(\dot{\phi}-2 \Omega_{s 1}\right) \\
& \times \sin \psi_{1} \cos ^{2} \frac{\psi_{1}}{2} e^{2 i \Omega_{s 1} t-i \phi},
\end{aligned}
$$

where we have ignored the $\exp [+i \phi]$ part of a driving force that does not give rise to resonance, since its effect is negligible. On the rhs of Eq. (17), we use $I_{22}^{r}$ to denote the $(2,2) r$-mode overlap. For Newtonian NS, $I_{22}^{r}$ is given by

$$
I_{22}^{r}=\sqrt{\frac{1}{m_{1} R_{1}^{4}} \int_{0}^{R_{1}} \rho r^{6} d r .}
$$

This is an analog of the tidal Love number for the $f$-mode.

In Eq. (17), $\psi_{1}$ is treated as a constant, because for a typical BNS system this angle changes on a timescale of $\sim 70 \mathrm{~s}$, which is much longer than the duration of resonance $(\sim 0.52 \mathrm{~s})$ and the time to merger ( $\sim \mathrm{s})$; see Appendix A for more details.

From Eq. (17), we can obtain the resonant condition in terms of the orbital frequency $\dot{\phi}_{r}$,

$$
\dot{\phi}_{r}=2 \Omega_{s 1}+\omega_{22}=\frac{4}{3} \Omega_{s 1},
$$

where we have used Eq. (13) to replace the mode frequency with the spin frequency. Note that the driving force is proportional to $\sim \sin \psi_{1} \cos ^{2} \psi_{1} / 2$; therefore, no $r$-mode 
DT takes place for $\psi_{1}=0, \pi$ when spins are aligned or antialigned with the orbital angular momentum.

\section{Gravitomagnetic coupling: Orbital part}

Let us now use the standard osculating equations to study the orbital evolution. Assume that there is a perturbing force $\boldsymbol{F}$ on the Newtonian binary system, which takes the form of

$$
\frac{\boldsymbol{F}}{\mu}=\mathcal{S} \lambda
$$

where $\mu$ is the reduced mass and $\lambda$ is the unit azimuthal vector in the orbital plane. The evolution of orbital separation $D(t)$ is given by [85]

$$
\frac{d D}{d t}=2 \sqrt{\frac{D^{3}}{M}} \mathcal{S} .
$$

In our case, $\mathcal{S}$ contains two parts. The first is the backreaction force due to $\mathrm{GW}$ radiation, given by the averaged (over orbital timescale) Burke-Thorne dissipation term [85]:

$$
\mathcal{S}_{\mathrm{GW}}=-\frac{32}{5} \eta\left(\frac{M}{D}\right)^{3} \sqrt{\frac{M}{D^{3}}} .
$$

The other part is the stellar induced force. The current quadrupole moment $J_{i j}$ induced by the $r$-mode (to the linear order in $\xi_{22}$ ) is given by ${ }^{3}$ [72]

$$
J_{i j} \sim I_{22}^{r} m_{1} R_{1}^{3} \omega_{22} e^{-2 i \Omega_{s 1} t} c_{22}+\text { c.c. },
$$

leading to an azimuthal acceleration $\mathcal{S}_{\text {tide }}$ [83]:

$$
\begin{aligned}
\mathcal{S}_{\text {tide }}= & \frac{8}{3} \sqrt{\frac{\pi}{5}} I_{22}^{r} \frac{\Omega_{s 1}\left(\omega_{22}+2 \Omega_{s 1}\right)}{D^{3}} M R_{1}^{3} \sin \psi_{1} \cos ^{2} \frac{\psi_{1}}{2} \\
& \times 2 \Re\left(-i e^{i \phi-2 i \Omega_{s} t} c_{22}\right) .
\end{aligned}
$$

The total acceleration is given by

$$
\mathcal{S}=\mathcal{S}_{\text {tide }}+\mathcal{S}_{\mathrm{GW}}
$$

Note that the current quadrupole moment also induces forces along the radial axis and along the axis of orbital angular momentum. These will lead to the evolution in orbital eccentricity and semilatus rectum. However, as argued in Ref. [72], these effects are negligible, and the orbit keeps quasicircular within the entire evolution process. We have also confirmed this numerically. Here we do not include those effects.

\footnotetext{
${ }^{3}$ This is different from the $f$-mode dynamical tides, where the leading term is the mass quadrupole moment.
}

Thus far we have considered only the $r$-mode of $m_{1}$. The resonance for $m_{2}$ can be treated using a direct replacement $(1 \leftrightarrow 2)$. Since we consider only the $(2,2) r$-mode in the two stars, we will drop the labels of $(l, m)$ and add a new subscript to refer to an individual star. For example, $I_{1}^{r}$ and $\omega_{1}$ stand for the $r$-mode overlap and frequency of $m_{1}$, respectively.

Combining Eqs. (17), (21), (22), and (24), we finally arrive at a complete set of EOMs for the binary system,

$\frac{d \phi}{d t}=\sqrt{\frac{M}{D^{3}}}$,

$$
\begin{aligned}
\frac{d D}{d t}= & -\frac{64}{5} \eta\left(\frac{M}{D}\right)^{3} \\
& +\frac{16}{3} \sqrt{\frac{\pi}{5}} I_{1}^{r} \frac{\Omega_{s 1}\left(\omega_{1}+2 \Omega_{s 1}\right)}{D^{3 / 2}} M^{1 / 2} R_{1}^{3} \\
& \times 2 \sin \psi_{1} \cos ^{2} \frac{\psi_{1}}{2} \Re\left(-i e^{i \phi-2 i \Omega_{s 1} t} c_{1}\right) \\
& +\frac{16}{3} \sqrt{\frac{\pi}{5}} I_{2}^{r} \frac{\Omega_{s 2}\left(\omega_{2}+2 \Omega_{s 2}\right)}{D^{3 / 2}} M^{1 / 2} R_{2}^{3} \\
& \times 2 \sin \psi_{2} \cos ^{2} \frac{\psi_{2}}{2} \Re\left(-i e^{i \phi-2 i \Omega_{s 2} t} c_{2}\right), \\
\dot{c}_{1}= & -i \omega_{1} c_{1}+\frac{i}{\omega_{1}} \sqrt{\frac{16 \pi}{5}} \frac{I_{1}^{r} m_{2} R_{1}}{D^{2}} \dot{\phi}\left(\dot{\phi}-2 \Omega_{s 1}\right) \\
& \times \sin \psi_{1} \cos ^{2} \frac{\psi_{1}}{2} e^{2 i \Omega_{s 1} t-i \phi}, \\
\dot{c}_{2}= & -i \omega_{2} c_{2}+\frac{i}{\omega_{2}} \sqrt{\frac{16 \pi}{5}} \frac{I_{2}^{r} m_{1} R_{2}}{D^{2}} \dot{\phi}\left(\dot{\phi}-2 \Omega_{s 2}\right) \\
& \times \sin \psi_{2} \cos ^{2} \frac{\psi_{2}}{2} e^{2 i \Omega_{s 2} t-i \phi} .
\end{aligned}
$$

By simultaneously integrating the stellar and orbital parts, we can obtain the evolution of the system and the gravitational waves that it emits.

\section{DYNAMICS OF $r$-MODE EXCITATION}

In this section, we discuss the features of $r$-mode excitation and its backaction onto orbital motion. In Sec. IVA, we first cast equations of motion (26) into forms that depend only on independent parameters, then provide the appropriate initial conditions. In Sec. IV B, we analytically describe orbital motion across tidal resonance and compare it to the results in FR07. In Sec. IV C, we provide analytical formulas for tidal excitation.

\section{A. Equation of motion}

Although Eqs. (26) are complete and can be integrated straightforwardly, it is difficult to see the actual dependence of solutions on specific parameters. For example, it is well known that the inspiraling process of a binary system is 
controlled by the chirp mass $\mathcal{M}=M \eta^{3 / 5}$ at the leading order, since the direct observable is the GW phase as a function of frequency. However, both the total mass $M$ and the symmetric mass ratio $\eta$ appear in the equations. The degeneracy between them is hidden. This problem can be fixed by rescaling the parameters in the following way:

$$
\begin{aligned}
& c_{1}=\bar{c}_{1} \frac{I_{1}^{r} m_{2} R_{1}}{\omega_{1} M^{2 / 3}} \sin \psi_{1} \cos ^{2} \frac{\psi_{1}}{2}, \\
& c_{2}=\bar{c}_{2} \frac{I_{2}^{r} m_{1} R_{2}}{\omega_{2} M^{2 / 3}} \sin \psi_{2} \cos ^{2} \frac{\psi_{2}}{2},
\end{aligned}
$$

and we replace the separation $D$ with the orbital frequency $\dot{\phi}$. In terms of these new parameters, the equations of motion become

$$
\begin{aligned}
\frac{d \dot{\phi}}{d t}= & \frac{96}{5} \mathcal{M}^{5 / 3} \dot{\phi}^{11 / 3}+32 \sqrt{\frac{\pi}{5}} \mathcal{I}_{1} \Omega_{s 1} \dot{\phi}^{8 / 3} \mathfrak{R}\left(-i e^{i \phi-2 i \Omega_{s 1} t} \bar{c}_{1}\right) \\
& +32 \sqrt{\frac{\pi}{5}} \mathcal{I}_{2} \Omega_{s 2} \dot{\phi}^{8 / 3} \mathfrak{R}\left(-i e^{i \phi-2 i \Omega_{s 2} t} \bar{c}_{2}\right), \\
\dot{\bar{c}}_{1}- & \frac{2}{3} i \Omega_{s 1} \bar{c}_{1}=i \sqrt{\frac{16 \pi}{5}}\left(\dot{\phi}-2 \Omega_{s 1}\right) \dot{\phi}^{7 / 3} e^{2 i \Omega_{s 1} t-i \phi}, \\
\dot{\bar{c}}_{2}- & \frac{2}{3} i \Omega_{s 2} \bar{c}_{2}=i \sqrt{\frac{16 \pi}{5}}\left(\dot{\phi}-2 \Omega_{s 2}\right) \dot{\phi}^{7 / 3} e^{2 i \Omega_{s 2} t-i \phi} .
\end{aligned}
$$

Here we have defined the $r$-mode coupling coefficient for each NS as

$$
\mathcal{I}_{i}=\bar{I}_{i}^{r 2} m_{i}^{4} \sin ^{2} \psi_{i} \cos ^{4} \frac{\psi_{i}}{2}\left(1-\frac{m_{i}}{M}\right)
$$

with the normalized $r$-mode overlap $\bar{I}^{r}$ defined by

$$
\bar{I}^{r}=\sqrt{\frac{1}{m_{\mathrm{NS}}^{5}} \int_{0}^{R_{\mathrm{NS}}} \rho r^{6} d r},
$$

where $m_{\mathrm{NS}}$ and $R_{\mathrm{NS}}$ are the mass and the radius of the NS. In Eqs. (29), $\mathcal{I}_{i}$ characterizes the tidal backreaction of NS $i$ onto the orbit. Note that $\mathcal{I}$ (the effect of the $r$-mode DT) vanishes when $\psi=0, \pi$ and is maximized when $\psi=\pi / 3$.

The initial conditions for Eqs. (29) were chosen such that the orbit is quasicircular and the tides are in equilibrium:

$$
\begin{aligned}
& \dot{\phi}^{(0)}=2 \pi F_{0}, \quad \phi^{(0)}=0, \\
& \bar{c}_{1}^{(0)}=\left(2 \pi F_{0}\right)^{4 / 3} \sqrt{\frac{16 \pi}{5}} \frac{\left(2 \pi F_{0}\right)^{2}-4 \Omega_{s 1} \pi F_{0}}{\frac{4}{3} \Omega_{s 1}-2 \pi F_{0}}, \\
& \bar{c}_{2}^{(0)}=\left(2 \pi F_{0}\right)^{4 / 3} \sqrt{\frac{16 \pi}{5}} \frac{\left(2 \pi F_{0}\right)^{2}-4 \Omega_{s 2} \pi F_{0}}{\frac{4}{3} \Omega_{s 2}-2 \pi F_{0}} .
\end{aligned}
$$

Written in this way, the EOM (as well as the initial conditions) depend on five parameters of the binary system: $\mathcal{M}, \mathcal{I}_{i}, \Omega_{s i}$, with $i=1,2$.

In the rest of this section, we shall numerically integrate the EOM and discuss features of the solutions. For example, we choose a $(1.4,1.4) M_{\odot}$ BNS system, with one of the stars spinning at frequency $\Omega_{s 1}=2 \pi \times 30 \mathrm{~Hz}$ and the other one nonspinning. We choose an inclination angle $\psi_{1}$ of $\pi / 3$. The EOS is polytopic with $\Gamma=2$, and the radius of both stars is chosen as $R_{\mathrm{NS}}=13 \mathrm{~km}$. This gives an $r$-mode overlap of $I_{1}^{r}=0.185$. There is only one resonance during the inspiral, and its resonant orbital frequency is given by

$$
\dot{\phi}\left(t_{r}\right)=\frac{4}{3} \Omega_{s}=2 \pi \times 40 \mathrm{~Hz} .
$$

We set an initial orbital frequency $F_{0}$ at $4.5 \mathrm{~Hz}$ and evolve the system up to the contact separation of $r^{\text {stop }}=2 R_{\mathrm{NS}}$.

\section{B. Orbital evolution}

As argued in FR07, the pre- and postresonance orbit can be well approximated as two PP orbits, with the orbital phase given as

$$
\phi^{(\mathrm{ana})}(t)= \begin{cases}\phi_{\mathrm{PP}}^{(\mathrm{pre})}(t) & \text { if } t<t_{r} \\ \phi_{\mathrm{PP}}^{(\mathrm{post})}(t) & \text { if } t>t_{r}\end{cases}
$$

$\phi^{\text {(post,pre) }}(t)$ both evolve without being affected by tides, following the same equation:

$$
\frac{d}{d t} \dot{\phi}^{\text {(post,pre })}=\frac{96}{5} \mathcal{M}^{5 / 3}\left[\dot{\phi}^{(\text {post,pre })}\right]^{11 / 3} .
$$

The initial condition for preresonance orbit is simply (the same as that used for numerical integration)

$$
\phi^{(\mathrm{pre})}(t=0)=0, \quad \dot{\phi}^{(\mathrm{pre})}(t=0)=2 \pi F_{0},
$$

and the resonance orbital frequency is given by

$$
\dot{\phi}_{\mathrm{PP}}^{(\mathrm{pre})}\left(t_{r}\right)=\frac{4}{3} \Omega_{s 1} \text {. }
$$

At resonance, $\phi_{\mathrm{PP}}^{(\mathrm{post})}\left(t_{r}\right)$ is related to $\phi_{\mathrm{PP}}^{(\mathrm{pre})}\left(t_{r}\right)$ with continuity in value and a jump in derivative, and one can write

$$
\begin{gathered}
\phi_{\mathrm{PP}}^{\mathrm{post}}\left(t_{r}\right)=\phi_{\mathrm{PP}}^{\mathrm{pre}}\left(t_{r}\right), \\
\Delta \dot{\phi}_{\text {tide }}=\dot{\phi}_{\mathrm{PP}}^{(\mathrm{post})}\left(t_{r}\right)-\dot{\phi}_{\mathrm{PP}}^{(\mathrm{pre})}\left(t_{r}\right)=\ddot{\phi}_{\mathrm{PP}}^{(\mathrm{pre})}\left(t_{r}\right) \delta t_{r} .
\end{gathered}
$$

In fact, Eqs. (37) and (38) can be grouped into a compact form [see Eq. (1.6) in FR07] 


$$
\phi_{\mathrm{PP}}^{\mathrm{post}}(t)=\phi_{\mathrm{PP}}^{\mathrm{pre}}\left(t+\delta t_{r}\right)-\delta \phi_{r}
$$

with [see Eq. (5.37) in FR07]

$$
\begin{aligned}
\delta t_{r}= & -\frac{5 \pi^{2}}{192}\left(\frac{4}{3}\right)^{-1 / 3} \frac{\Omega_{s 1}^{-1 / 3}}{\mathcal{M}^{10 / 3}} \mathcal{I}_{1} \\
= & -2.64 \times 10^{-4} \mathrm{~s}\left(\frac{\Omega_{s 1}}{2 \pi \times 30 \mathrm{~Hz}}\right)^{-1 / 3}\left(\frac{\sin ^{2} \psi_{1} \cos ^{4} \psi_{1} / 2}{0.422}\right) \\
& \times\left(\frac{m_{1}}{1.4 M_{\odot}}\right)^{3}\left(\frac{M}{2.8 M_{\odot}}\right)^{-7 / 3}\left(\frac{\eta}{0.25}\right)^{-1}\left(\frac{\bar{I}_{1}^{r}}{7.32}\right)^{2} \\
\delta \phi_{r}= & \dot{\phi}\left(t_{r}\right) \delta t_{r}=-\frac{5 \pi^{2}}{192}\left(\frac{4}{3}\right)^{2 / 3} \frac{\Omega_{s 1}^{2 / 3}}{\mathcal{M}^{10 / 3}} \mathcal{I}_{1} \\
= & -6.65 \times 10^{-2} \operatorname{rad}\left(\frac{\Omega_{s 1}}{2 \pi \times 30 \mathrm{~Hz}^{2 / 3}}\right)^{2 / 3}\left(\frac{M_{1}}{2.8 M_{\odot}}\right)^{-7 / 3} \\
& \times\left(\frac{\sin ^{2} \psi_{1} \cos ^{4} \psi_{1} / 2}{0.422}\right)^{-1}\left(\frac{m_{1}}{1.4 M_{\odot}}\right)^{2}\left(\frac{\eta}{7.32}\right)^{r} \cdot \\
& \times\left(\frac{\eta}{0.25}\right)^{-1}(40 \mathrm{~b})
\end{aligned}
$$

Here $\delta t_{r}$ and $\delta \phi_{r}$ are the effective orbital time and phase shifts between the pre- and postresonance PP orbits.

Note that $\Delta \dot{\phi}_{\text {tide }}<0$ and $\dot{\phi}_{\mathrm{PP}}^{(\mathrm{post})}\left(t_{r}\right)<\dot{\phi}_{\mathrm{PP}}^{(\mathrm{pre})}\left(t_{r}\right)$. The resonance transfers the energy from star to orbit, which is different from the case of the $f$-mode (see Fig. 8 in Ref. [53]). This can be understood using the relation $[71,72]$

$$
\begin{aligned}
\Delta E_{\mathrm{star}}= & \frac{E_{\text {mode }}}{\omega_{l m}}\left(\omega_{l m}+m \Omega_{s 1}\right) \\
= & -9.9 \times 10^{46} \operatorname{erg}\left(\frac{\Omega_{s 1}}{2 \pi \times 30 \mathrm{~Hz}}\right)^{3}\left(\frac{\bar{I}_{1}^{r}}{7.32}\right)^{2} \\
& \times\left(\frac{m_{1}}{1.4 M_{\odot}}\right)^{4}\left(\frac{\sin ^{2} \psi_{1} \cos ^{4} \psi_{1} / 2}{0.422}\right)\left(\frac{1-\Xi}{0.5}\right),
\end{aligned}
$$

where $E_{\text {mode }}=b_{l m} \omega_{l m}\left|c_{l m}\right|^{2}>0$ and $\Delta E_{\text {star }}$ is the change of stellar energy. Therefore, for any mode that is subjected to the Chandrasekhar-Friedman-Schutz instability condition $[86,87]$

$$
\omega_{l m}\left(\omega_{l m}+m \Omega_{s 1}\right)<0
$$

the tidal excitation leads to the loss of stellar energy and the decrease of spin frequency. ${ }^{4}$ For completeness, the angular momentum transfer can be written as

\footnotetext{
${ }^{4}$ For a given baryon number and total angular momentum, the uniform angular velocity is the minimum-energy state $[88,89]$.
}

$$
\begin{aligned}
\Delta L_{\text {star }}= & \frac{E_{\text {mode }}}{\omega_{l m}} \\
= & -3.9 \times 10^{44} \mathrm{erg} \cdot \mathrm{s}\left(\frac{\Omega_{s 1}}{2 \pi \times 30 \mathrm{~Hz}}\right)^{2}\left(\frac{\bar{I}_{1}^{r}}{7.32}\right)^{2} \\
& \times\left(\frac{m_{1}}{1.4 M_{\odot}}\right)^{4}\left(\frac{\sin ^{2} \psi_{1} \cos ^{4} \psi_{1} / 2}{0.422}\right)\left(\frac{1-\Xi}{0.5}\right)
\end{aligned}
$$

and the change of spin frequency is given by

$$
\begin{aligned}
\Delta \Omega_{s}= & -2 \pi \times 3.6 \times 10^{-2} \mathrm{~Hz}\left(\frac{\Omega_{s 1}}{2 \pi \times 30 \mathrm{~Hz}}\right)^{2}\left(\frac{\bar{I}_{1}^{r}}{7.32}\right)^{2} \\
& \times\left(\frac{m_{1}}{1.4 M_{\odot}}\right)^{4}\left(\frac{\sin ^{2} \psi_{1} \cos ^{4} \psi_{1} / 2}{0.422}\right) \\
& \times\left(\frac{1-\Xi}{0.5}\right)\left(\frac{\bar{I}}{14.6}\right)^{-1}
\end{aligned}
$$

which is negligible in our study.

Using the above binary system, we first investigate how the numerical integration of the EOM deviates from $\dot{\phi}_{\mathrm{PP}}^{(\mathrm{pre})}(t)$. In Fig. 3, we plot $\dot{\phi}_{\mathrm{PP}}^{(\mathrm{pre})}(t)-\dot{\phi}^{(\text {num })}(t)$ as a black line. The vertical, blue-dashed line stands for the time of resonance. In the preresonance regime $t<t_{r},\left|\Delta \dot{\phi}_{\text {tide }}\right|<$ $2 \pi \times 10^{-4} \mathrm{~Hz}$ and is contributed mainly by the adiabatic tide. During the resonance, $\left|\Delta \dot{\phi}_{\text {tide }}\right|$ quickly grows to $2 \pi \times 10^{-3} \mathrm{~Hz}$. Then, in the postresonance regime, there is a small oscillation on top of the major deviation, which is caused by the $r$-mode oscillation. We also saw this feature in the case of the $f$-mode [53]. Note that $\dot{\phi}^{(\text {num })} \sim 2 \pi \times$ $10^{2}-2 \pi \times 10^{3} \mathrm{~Hz}$ for the time interval that we present. The deviation caused by the resonance is extremely small.

Equations (40) show that typical values for $\delta \phi_{r}$ and $\delta t_{r}$ are $-6.65 \times 10^{-2} \mathrm{rad}$ and $-2.64 \times 10^{-4} \mathrm{~s}$, respectively. The induced change in orbital frequency $\left|\Delta \dot{\phi}_{\text {tide }}\left(t_{r}\right)\right|$ is $2 \pi \times 10^{-3} \mathrm{~Hz}$, which corresponds to $\mathrm{P}$ in Fig. 3 . With $\mathrm{P}$ as a new initial condition, we obtain $\dot{\phi}_{\mathrm{PP}}^{\text {(post) }}(t)$ by solving Eq. (34). In Fig. 3, we show $\dot{\phi}_{\mathrm{PP}}^{(\text {pre })}(t)-\dot{\phi}_{\mathrm{PP}}^{(\text {post })}(t)$ as a red dashed line. We can see that $\dot{\phi}_{\mathrm{PP}}^{\text {(post) }}(t)$ tracks the averaged $\dot{\phi}^{(\text {num })}(t)$ well in the postresonance regime.

\section{Tidal evolution}

Let us move on to the stellar part. Following the procedure used in Sec. III of Ref. [53], we define two real-valued quadratures, $A$ and $B$, from the $r$-mode amplitude,

$$
A+i B=\bar{c}_{1} e^{i \phi-i m \Omega_{s 1} t} \quad \text { and } \quad m=2,
$$

with $A$ determining the radial force and $B$ the torque backreacting onto the orbit, respectively. Here $m=2$ since 


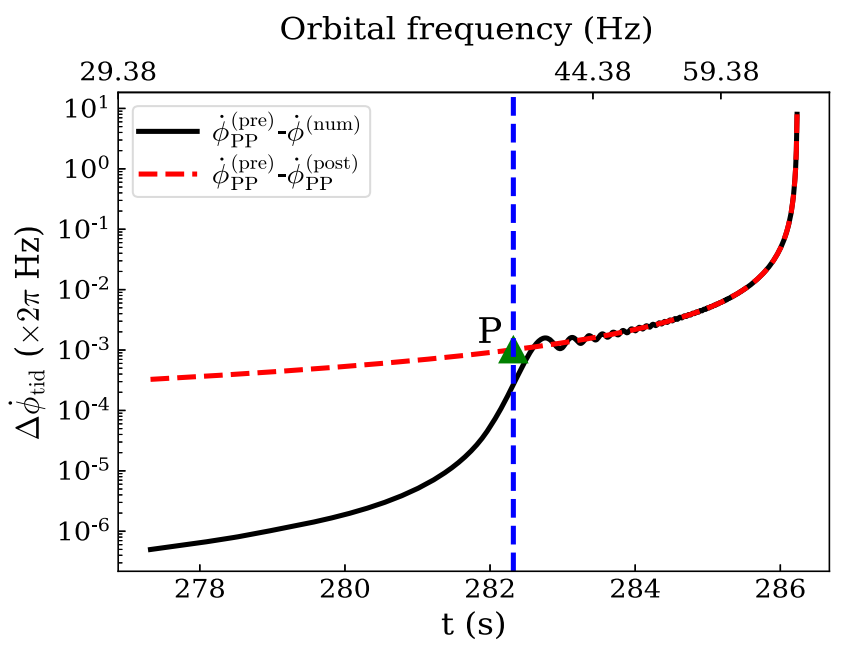

FIG. 3. The orbital dynamics near the $r$-mode resonance. The BNS system has individual masses $(1.4,1.4) M_{\odot}$. One of them spins at $\Omega_{s 1}=30 \mathrm{~Hz}$ with the inclination angle $\psi_{1}=\pi / 3$, whereas the other one is nonspinning. The EOS is $\Gamma=2$ polytrope with radius $R_{\mathrm{NS}}=13 \mathrm{~km}$. The vertical dashed line represents the time of resonance $t_{r}$. The preresonance PP orbit $\dot{\phi}_{\mathrm{PP}}^{(\mathrm{pre})}(t)$, which has the same initial condition as the numerical one, is compared to the numerical integration, as shown by the black curve. Before the resonance, $\left|\Delta \dot{\phi}_{\text {tide }}\right|$ is below $\sim 2 \pi \times 10^{-4} \mathrm{~Hz}$, which is caused mainly by the adiabatic $r$-mode. After the resonance, there are some oscillatory features, which are from the $r$-mode oscillation. Equation (38) gives the new orbital frequency after the $r$-mode is excited, which is labeled "P" in the figure. Using $\mathrm{P}$ as a new initial condition, we obtain the other PP orbit $\dot{\phi}_{\mathrm{PP}}^{\text {(post) }}(t)$. The difference between $\dot{\phi}_{\mathrm{PP}}^{(\text {pre })}(t)$ and $\dot{\phi}_{\mathrm{PP}}^{\text {(post) }}(t)$ is shown as a red dashed line, which tracks the averaged numerical result very well.

we are focusing on the $(l=2, m=2)$ mode. Using integration by parts, we obtain analytic expressions of $A$ and $B$ as

$$
\begin{aligned}
& A=-\dot{\phi}^{7 / 3} \sqrt{\frac{16 \pi}{5}} \frac{\left(2 \Omega_{s 1}-\dot{\phi}\right)}{-\dot{\phi}+\frac{4}{3} \Omega_{s 1}} \\
& +\frac{\dot{\phi}_{r}^{10 / 3}}{\sqrt{\dot{\Omega}_{r}}} \sqrt{\frac{4 \pi}{5}}\left[\sqrt{\pi} F C\left(\frac{\hat{t}}{\sqrt{\pi}}\right) \sin \Theta+\sqrt{\frac{\pi}{2}} \sin \left(\Theta-\frac{\pi}{4}\right)\right. \\
& \left.-\sqrt{\pi} F S\left(\frac{\hat{t}}{\sqrt{\pi}}\right) \cos \Theta-\frac{\cos \left(\Theta-\frac{1}{2} \hat{t}^{2}\right)}{\hat{t}}\right] \text {, } \\
& B=-\frac{\dot{\phi}_{r}^{10 / 3}}{\sqrt{\dot{\Omega}_{r}}} \sqrt{\frac{4 \pi}{5}}\left[\sqrt{\pi} F C\left(\frac{\hat{t}}{\sqrt{\pi}}\right) \cos \Theta+\sqrt{\frac{\pi}{2}} \cos \left(\Theta-\frac{\pi}{4}\right)\right. \\
& \left.+\sqrt{\pi} F S\left(\frac{\hat{t}}{\sqrt{\pi}}\right) \sin \Theta+\frac{\sin \left(\Theta-\frac{1}{2} \hat{t}^{2}\right)}{\hat{t}}\right] \text {. }
\end{aligned}
$$

where [Eq. (34)]
Orbital frequency $(\mathrm{Hz})$

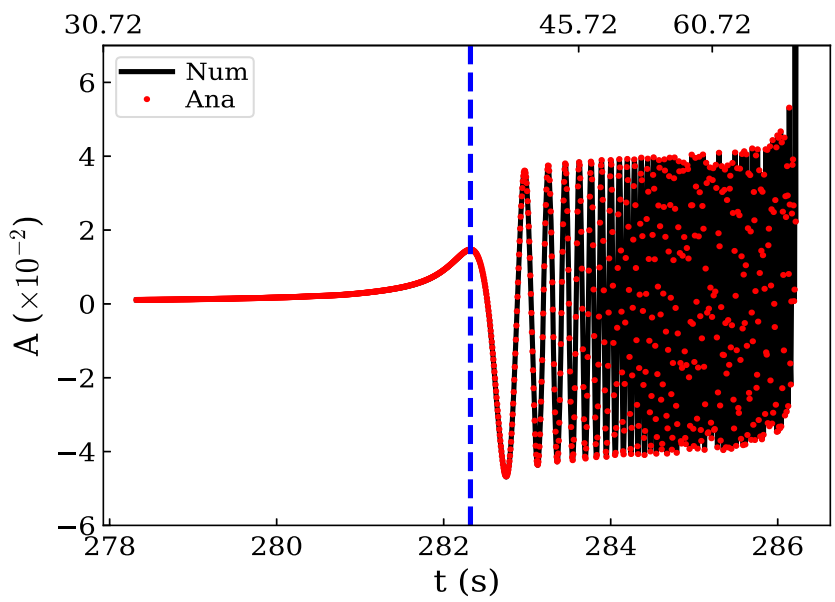

Orbital frequency $(\mathrm{Hz})$

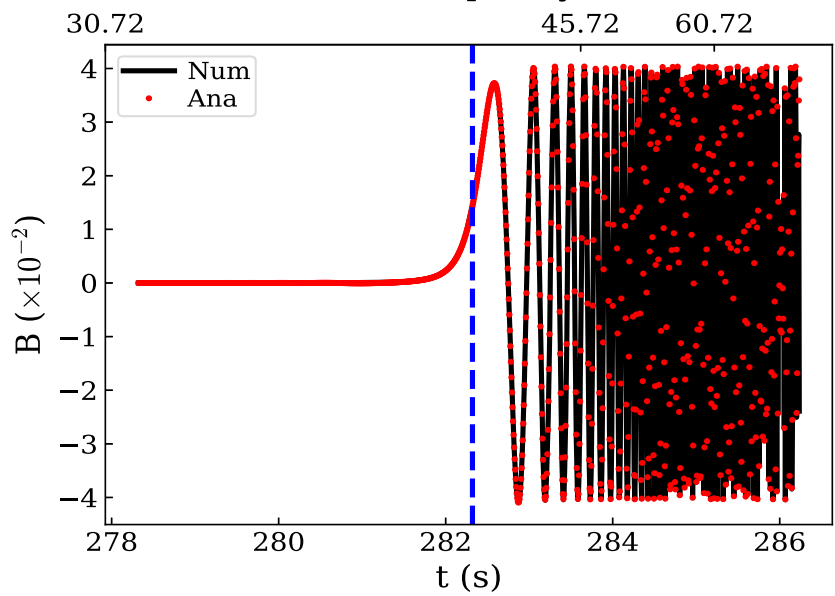

FIG. 4. Time evolution of $r$-mode amplitudes, $A$ (upper panel) and $B$ (lower panel). The black curves are from the numerical integration of Eqs. (29), and the red dots are from our analytic approximations in Eqs. (46). The vertical dashed line is the time of resonance from the numerical simulation. Our analytic results agree with the numerical ones to high accuracy. Unlike the $f$-mode, the variable $A$ diverges as the two NSs become close to each other. This is caused by differences in the adiabatic tide [see the first line of Eq. (46a)].

$$
\begin{aligned}
\frac{1}{\dot{\Omega}_{r}^{1 / 2}} & =\left[\frac{96}{5} \mathcal{M}^{5 / 3}\left(\frac{4}{3} \Omega_{s 1}\right)^{11 / 3}\right]^{-1 / 2} \\
& =0.2 \mathrm{~s}\left(\frac{\mathcal{M}}{1.22 M_{\odot}}\right)^{-5 / 6}\left(\frac{\Omega_{s 1}}{2 \pi \times 30 \mathrm{~Hz}}\right)^{-11 / 6} .
\end{aligned}
$$

The phases of $A$ and $B$ in the postresonance regime is controlled by two quantities,

$$
\begin{aligned}
& \Theta=\chi_{r}-\omega_{1} t+\phi-2 \Omega_{s 1} t, \\
& \hat{t}=\sqrt{\dot{\Omega}_{r}}\left(t-t_{r}\right),
\end{aligned}
$$

with the constant defined by $\chi_{r}=\omega_{1} t_{r}-\phi_{r}+2 \Omega_{s 1} t_{r}$, which results in $\Theta_{r}=0$ on resonance. Note that $1 / \sqrt{\dot{\Omega}_{r}} \sim 0.2 \mathrm{~s}$ 


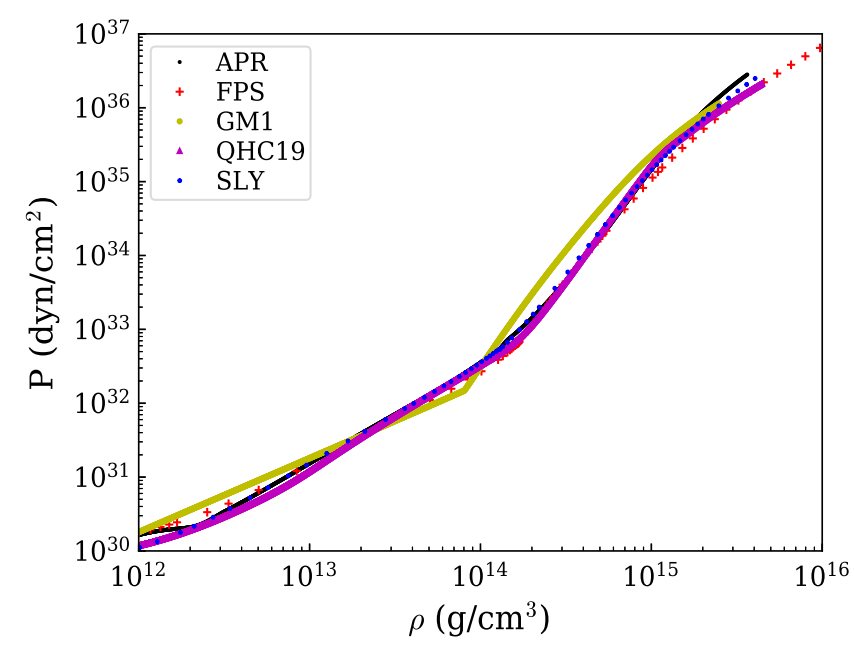

FIG. 5. Several EOSs for NSs used in this paper.

in Eq. (48b) is the duration of tidal excitation, which is much longer than the effective orbital time shift $\delta t_{r} \sim$ $10^{-4} \mathrm{~s}$ induced by the tide [Eq. (40a)].

In Fig. 4, we compare Eqs. (46) to numerical integration for the binary system mentioned above. We can see that our formulas are accurate in all regimes: adiabatic, resonance, and postresonance. The evolutions of $A$ and $B$ are similar to those of the $f$-mode [53], except the fact that $A$ increases toward infinity as the two stars become close to each other (recall that the amplitude of $A$ for the $f$-mode remains constant after the resonance). This can be easily understood from Eq. (46a). The first line of Eq. (46a), i.e., the adiabatic part, diverges as $\dot{\phi}^{7 / 3}$ when $\dot{\phi} \rightarrow \infty$. On the other hand, the term remains $\sim 1$ for the $f$-mode, which stays constant as the two stars come into contact. In spite of the diverging feature of $r$-mode in the late time evolution, our numerical

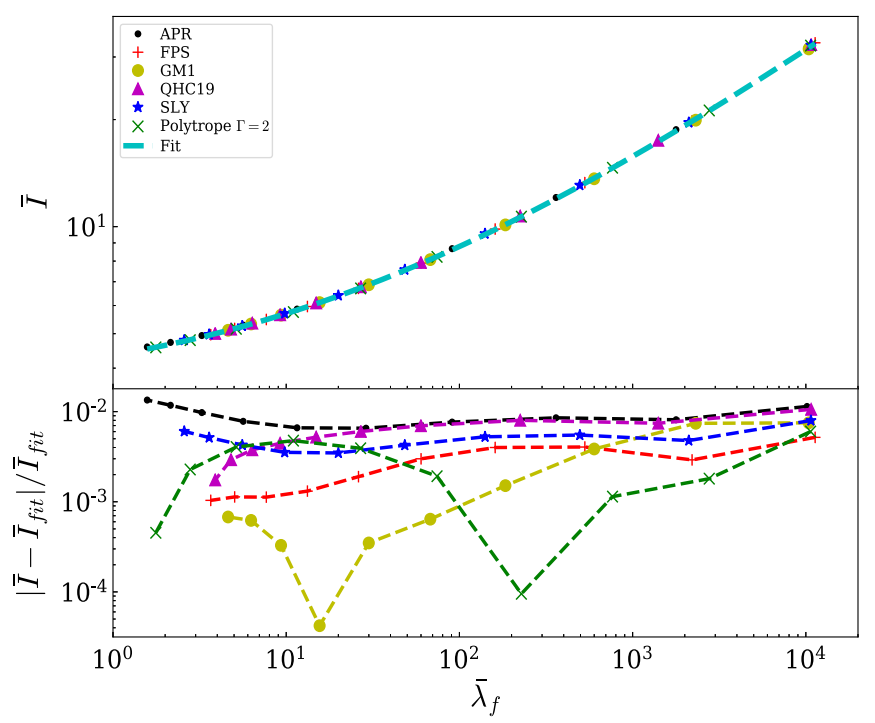

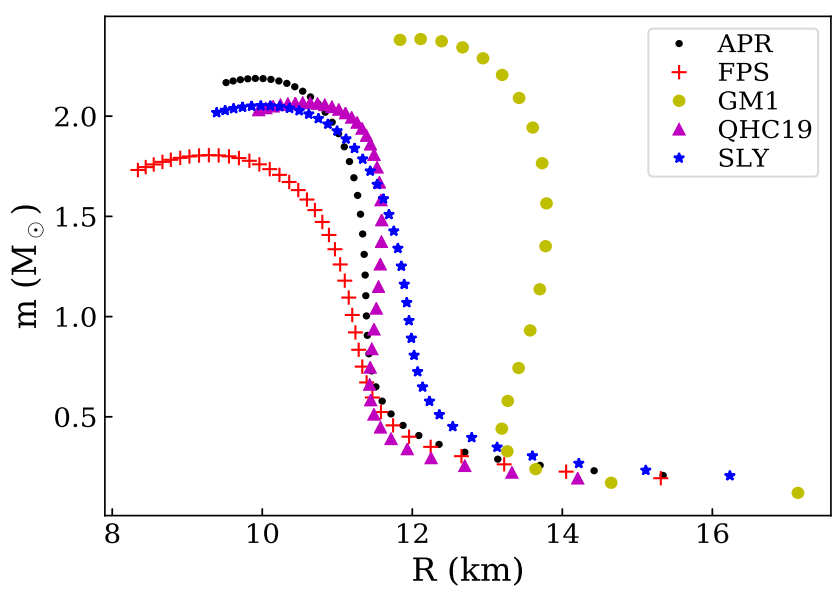

FIG. 6. NS mass-radius relation with different EOSs.

calculation shows that it does not lead to any detectable effect (for $3 \mathrm{G}$ detectors).

\section{ROSSBY-MODE OVERLAPS FOR DIFFERENT EQUATIONS OF STATE: A NEW UNIVERSAL RELATION}

In the last section, we saw that the effect of the $r$-mode enters into the EOM through the normalized overlap $\bar{I}^{r}$. Here we shall identify a new universal relation between $\bar{I}^{r}$ and the tidal Love number $\lambda_{f}$. We consider five realistic EOSs for cold NSs: APR [90], FPS [91], GM1 [92,93], QHC19 [94-96], and SLY [93], which are shown in Fig. 5. Among them, the data of FPS are from Ref. [97] and the rest are obtained from an EOS database, CompOSE [98]. For comparison, we also include a polytropic EOS with $P \propto \rho^{\Gamma}$ and $\Gamma=2$. The mass-radius relations from those EOSs are shown in Fig. 6.

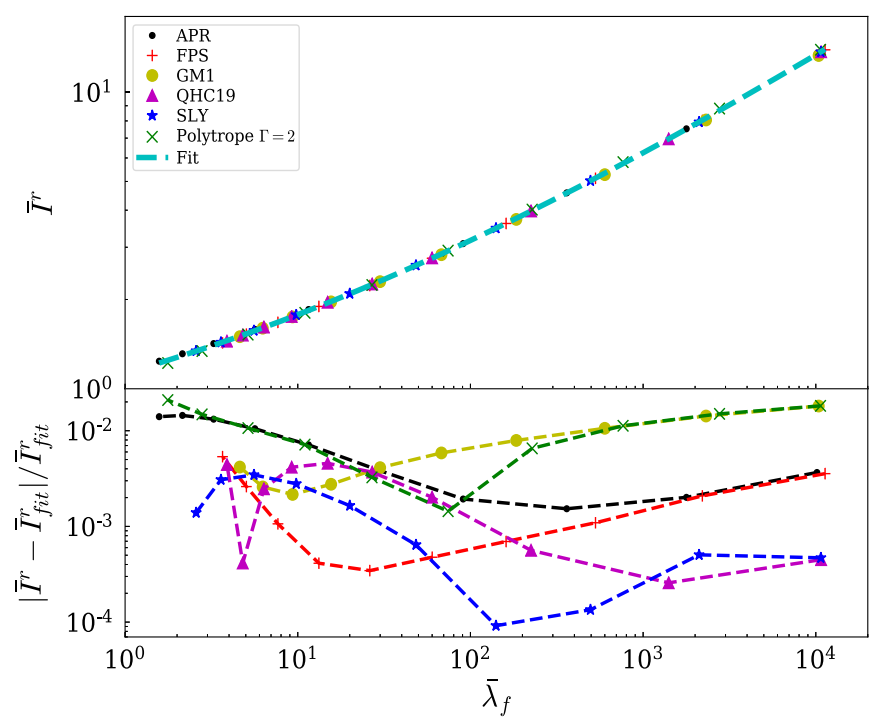

FIG. 7. The I-Love and $\bar{I}^{r}$-Love universal relations for several EOSs, as well as the fitting formulas in Eq. (52). The bottom two plots are fractional errors between true values and fitted results; errors of both relations are within $10^{-2}$ for $\bar{\lambda}_{f}$ ranging from $\mathcal{O}(1)$ to $\mathcal{O}\left(10^{4}\right)$. 
In Sec. VA, we first calculate $\bar{I}^{r}$ by solving TolmanOppenheimer-Volkoff (TOV) equations. Then, in Sec. V B, we explore the universal relation between $I^{r}$ and $\lambda_{f}$.

\section{A. The calculation of $I^{r}$}

We calculate $I^{r}$ at the zeroth order, i.e., using an unperturbed NS with spherical symmetry. Its metric is given by

$$
d s^{2}=-e^{\nu} d t^{2}+e^{\lambda} d r^{2}+r^{2}\left(d \theta^{2}+\sin ^{2} \theta d \phi^{2}\right),
$$

and we can obtain the density profile $\rho(r)$ by solving the TOV equations (see Appendix B for more details). We then perform a numerical integration to get $I^{r}$ based on its definition in Eq. (18). $\bar{I}^{r}$ is related to $I^{r}$ by

$$
I^{r}=\bar{I}^{r} \mathcal{C}^{2}
$$

where $\mathcal{C}$ is the compactness of the NS

$$
\mathcal{C}=\frac{m_{\mathrm{NS}}}{R_{\mathrm{NS}}}
$$

\section{B. Universal relations}

To establish the universal relation between the tidal Love number and the moment of inertia, Yagi and Yunes [77,78] normalized the tidal Love number $\lambda_{f}$ and the moment of inertia $I$ as $\bar{\lambda}_{f}=\lambda_{f} / m_{\mathrm{NS}}^{5}$ and $\bar{I}=I / m_{\mathrm{NS}}^{3}$. (The calculation of $\bar{\lambda}_{f}$ is summarized in Appendix C.) The universal relation between $\lambda_{f}$ and $\bar{I}$ is shown in the left panel of Fig. 7 .

Similarly, we plot $\bar{I}^{r}$ as functions of $\bar{\lambda}_{f}$ for various EOSs in the right panel of Fig. 7. We can see that their relation is also insensitive to the EOS. As Yagi and Yunes did, we fit the relation with a polynomial on a log-log scale

$$
\log y=a+b \log \bar{\lambda}_{f}+c\left(\log \bar{\lambda}_{f}\right)^{2}+d\left(\log \bar{\lambda}_{f}\right)^{3}+e\left(\log \bar{\lambda}_{f}\right)^{4},
$$

where $y=\bar{I}^{r}$ or $\bar{I}$. Results are shown in Table IV, where we also list the I-Love relation for comparison. In Fig. 7 we compare the fitted results to the true values. We can see that the relative errors for the two relations are similar and within $10^{-2}$.

Note that although the profile of $\rho$ comes from the solution of Einstein's equation (i.e., the TOV equations), our definition of $\bar{I}^{r}$ in Eq. (31) still used a Newtonian model of the NS and post-Newtonian equations for the gravitomagnetic coupling. We conjecture that a universal relation will still exist after relativistic corrections are made, but we anticipate systematic corrections to the form of the relation.
TABLE IV. Coefficients for the fitting formulas of the NS I-Love and $\bar{I}^{r}$-Love relations.

\begin{tabular}{lccccc}
\hline \hline$y$ & $a$ & $b$ & $c$ & $d$ & $e$ \\
\hline $\bar{I}^{r}$ & 0.121 & 0.169 & $1.25 \times 10^{-2}$ & $-9.38 \times 10^{-5}$ & $-1.92 \times 10^{-5}$ \\
$\bar{I}$ & 1.47 & $8.17 \times 10^{-2}$ & $1.49 \times 10^{-2}$ & $2.87 \times 10^{-4}$ & $-3.64 \times 10^{-5}$ \\
\hline \hline
\end{tabular}

\section{GRAVITATIONAL WAVES}

This section focuses on gravitational waves emitted by binaries that contain at least one spinning NS with $r$-mode resonance. In Sec. VI A, we construct a hybrid waveform model that incorporates both $r$-mode and PN effects. In Sec. VIB, we compare Flanagan and Racine's analytical formula for an $r$-mode-resonance-induced GW phase to the numerical results.

\section{A. A hybrid PN-r-mode waveform model}

Once we obtain the orbital evolution from the EOM, we can extract the numerical GW waveform through the (mass) quadrupole formula [85]

$$
h(t)=\frac{1}{D_{L}}\left(\ddot{Q}_{x x}-\ddot{Q}_{y y}\right),
$$

where $D_{L}$ is the distance between the detector and the source. Here we assume that the BNS is optimally oriented for the plus polarization. The quadrupole moment of the system is given by

$$
Q_{i j}=\mu\left(x_{i} x_{j}-r^{2} \delta_{i j} / 3\right),
$$

where only the orbital part is included because the $r$-mode does not induce an additional mass quadrupole moment. ${ }^{5}$

It is usually convenient to analyze data in the frequency domain (FD). Following Ref. [53], we first sample the numerical $h(t)$ in the time domain with a rate of $8192 \mathrm{~Hz}$, then use the fast Fourier transform algorithm to transform the data to FD. Finally, we select data in the frequency band $\left[2 F_{0}, 2 F_{\text {contact }}\right]$. (Note that at the mass quadrupole order, the GW frequency is twice the orbital frequency.)

Our numerical FD waveform $\tilde{h}^{N+r}(f)$ contains the leading-order PP contribution as well as the effect of $r$-mode resonance; its phase $\Psi_{N+r}$ can be written as

$$
\Psi_{N+r}=\Psi_{N}+\Psi_{r},
$$

where $\Psi_{r}$ is the phase induced by the $r$-mode resonance and $\Psi_{N}$ corresponds to the leading order of the PP waveform. With the stationary phase approximation (SPA), $\Psi_{N}$ can be written as

\footnotetext{
${ }^{5}$ The $r$-mode does contribute to gravitational radiation through the current quadrupole moment [Eq. (23)]. However, it is $10^{-7}$ smaller than the orbital mass quadrupole moment, which is negligible in our case.
} 


$$
\Psi_{N}=2 \pi f t_{c}-\phi_{c}+\frac{3}{128}(\pi \mathcal{M} f)^{-5 / 3}
$$

where $t_{c}$ and $\phi_{c}$ are the time and the GW phase of coalescence.

To incorporate other $\mathrm{PN}$ and ( $f$-mode) tidal effects into the waveform, we introduce a phase correction within the SPA framework, writing

$$
\tilde{h}(f)=\tilde{h}^{N+r}(f) e^{i \Psi_{\mathrm{SPA}}}
$$

The total phase of $\tilde{h}(f), \Psi_{\text {tot }}$, can be written as

$$
\Psi_{\mathrm{tot}}=\Psi_{N}+\Psi_{r}+\Psi_{\mathrm{SPA}}
$$

with

$$
\Psi_{\mathrm{SPA}}=\Psi_{\mathrm{PP}}+\Psi_{S O}+\Psi_{\bar{\lambda}_{f}^{(1)}}+\Psi_{\bar{\lambda}_{f}^{(2)}} .
$$

Here $\Psi_{\mathrm{PP}}$ is the PN correction to the phase of nonspinning $\mathrm{PP}$ up to $3.5 \mathrm{PN}^{6}$ [99], $\Psi_{S O}$ is the spin-orbit coupling term [100-103], and $\Psi_{\bar{\lambda}_{f}^{(1)}}$ and $\Psi_{\bar{\lambda}_{f}^{(2)}}$ are $f$-mode tidal effects from $m_{1}$ and $m_{2}$, respectively [104-106]. We have ignored the spin-spin coupling and the spin precession because their effects are negligible (see Appendix A). Expressions for the phase terms are shown in Appendix D.

Let us briefly review the parameter dependence of our waveforms. The Newtonian-plus- $r$-mode part of the waveform, $\tilde{h}^{N+r}$, depends on eight independent parameters: chirp mass $\mathcal{M}$, two individual spin frequencies $\Omega_{s i}$, two individual $r$-mode coupling coefficients $\mathcal{I}_{i}$, the luminosity distance $D_{L}$, and the coalescing time and phase $t_{c}$ and $\phi_{c}$. The phase correction, $\Psi_{\mathrm{SPA}}$, depends on five additional parameters: mass ratio $\Xi=m_{1} / M$, the (anti)symmetric tidal Love numbers $\bar{\lambda}_{f}^{s(a)}=\left(\bar{\lambda}_{f}^{1} \pm \bar{\lambda}_{f}^{2}\right) / 2$, and the (anti) symmetric dimensionless spin along the orbital angular momentum $\chi_{s(a)}^{(z)}=\left(\chi_{1}^{(z)} \pm \chi_{2}^{(z)}\right) / 2$. Here we use the normalized Love number (Sec. V B) because in this way it is more convenient to incorporate universal relations into the calculation.

\section{B. Analytic model for $\Psi_{r}$}

As discussed in Sec. IV B, DTs affect the orbital evolution significantly only near the resonance. In the postresonance regime, the orbit is well described by a PP orbit. Similarly, in the FD, resonance leads only to a phase shift $\delta \Phi_{r}$ and a time shift $\delta t_{r}$ to the waveform, i.e., [72]

$$
\Psi_{r}=\left(\delta \Phi_{r}-2 \pi f \delta t_{r}\right) \Theta\left(f-f_{r}\right),
$$

\footnotetext{
${ }^{6}$ This excludes the leading-order contribution, which is already contained in $\Psi_{N}$.
}

where the resonant GW frequency is given by [Eq. (19)]

$$
f_{r}=\frac{\dot{\phi}_{r}}{\pi}=\frac{4}{3 \pi} \Omega_{s 1}
$$

The Heaviside step function $\Theta\left(f-f_{r}\right)$ is introduced here because there is no DT when $f<f_{r} .{ }^{7}$ Using Eqs. (40b) and (40a) and the relation

$$
\delta \Phi_{r}=2 \delta \phi_{r}
$$

we obtain

$$
\delta t_{r}=\frac{\delta \Phi_{r}}{2 \pi f_{r}}
$$

we arrive at

$$
\Psi_{r}=\left(1-\frac{f}{f_{r}}\right) \delta \Phi_{r} \Theta\left(f-f_{r}\right)
$$

We refer the interested reader to Appendix B in Ref. [45] for a strict derivation. In Fig. 8, we compare Eq. (64) to the numerical result, using the same BNS system as Fig. 3 except that $\Omega_{s 1}=80 \mathrm{~Hz}$. We can see that the phase difference induced by the $r$-mode, $\Psi_{\mathrm{GW}}^{(\mathrm{num})}-\Psi_{\mathrm{GW}}^{(\mathrm{pre})}$, agrees well with the expression of $\Psi_{r}$ in Eq. (64). Here $\Psi_{\mathrm{GW}}^{(\mathrm{num})}$ stands for the real GW phase and $\Psi_{\mathrm{GW}}^{(\mathrm{pre})}$ is the GW phase of the preresonance PP orbit (extending to the postresonance regime).

The expression of $\Psi_{r}$ results in

$$
\begin{aligned}
& \frac{\partial \tilde{h}^{N+r}}{\partial \mathcal{I}} \sim \frac{\partial \tilde{h}^{N+r}}{\partial \delta \Phi_{r}}=i\left(1-\frac{f}{f_{r}}\right) \Theta\left(f-f_{r}\right) \tilde{h}^{N+r}, \\
& \frac{\partial \tilde{h}^{N+r}}{\partial \boldsymbol{\Omega}_{s}} \sim \frac{\partial \tilde{h}^{N+r}}{\partial f_{r}}+\frac{\partial \tilde{h}^{N+r}}{\partial \delta \Phi_{r}} \frac{\partial \delta \Phi_{r}}{\partial \Omega_{s 1}} \sim \frac{f}{f_{r}^{2}} \delta \Phi_{r} \Theta\left(f-f_{r}\right) \tilde{h}^{N+r},
\end{aligned}
$$

which are important to understanding the resulting Fisher analysis, as we will discuss in the next section. In Eq. (65b), we have ignored the term $\partial \tilde{h}^{N+r} / \partial \delta \Phi_{r}$ because it is suppressed by the factor $\left(1-f / f_{r}\right)$ when $f \sim f_{r}$.

We can learn two things from Eqs. (65). First, $\partial \tilde{h}^{N+r} / \partial \delta \mathcal{I}$ is proportional to $\left(1-f / f_{r}\right)$, which is close to 0 when $f \sim f_{r}$, so the constraint for $\mathcal{I}$ from GW detection is weaker than the one for $\Omega_{s}$. Second, the constraint for $\mathcal{I}$ is independent of the magnitude of $\delta \Phi_{r}$

\footnotetext{
${ }^{7}$ Here we assume that the preresonance PP orbit is aligned with the true orbit, so the phase and time shifts appear after the resonance. It is also equivalent to aligning the postresonance PP orbit with the real orbit. Then the Heaviside step function should be changed to $\Theta\left(f_{r}-f\right)$.
} 


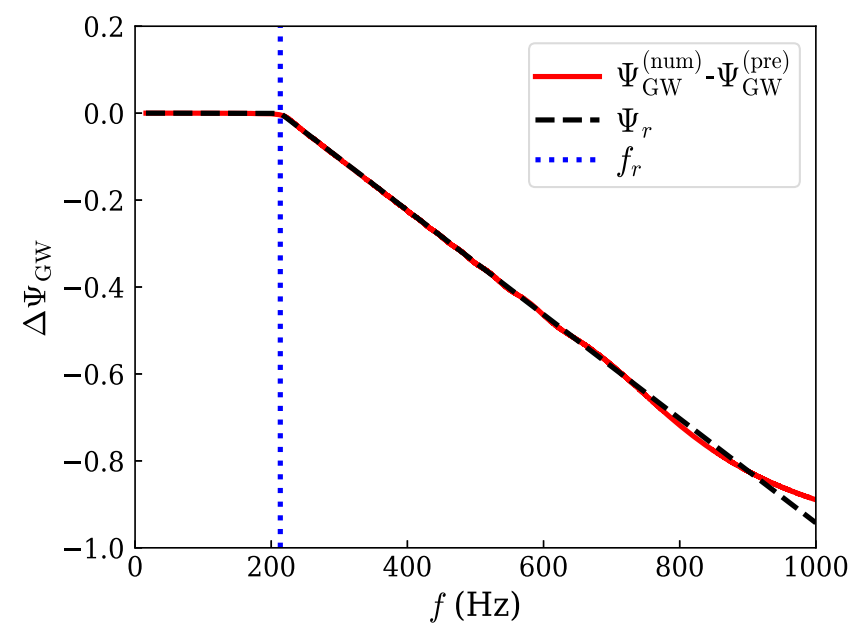

FIG. 8. The GW phase difference induced by the $r$-mode DT versus the GW frequency. This is compared to the expression of $\Psi_{r}$ in Eq. (64). The BNS system is the same as that used in Fig. 3 except that $\Omega_{s 1}=80 \mathrm{~Hz}$.

(or $\mathcal{I}$ ), while the constraint for $\Omega_{s}$ is proportional to $\delta \Phi_{r}^{-1} \sim$ $\mathcal{I}^{-1}$ (see also Ref. [45]).

\section{CONSTRAINTS OF PARAMETERS USING THE $r$-MODE DYNAMICAL TIDE}

In this section, we discuss parameter estimation based on the hybrid PN-r-mode waveform obtained in Sec. VIA.

\section{A. Fisher-matrix formalism and signal strength}

We shall do this by computing the Fisher information matrix, defined by

$$
\Gamma_{i j}=\left(\frac{\partial h}{\partial \theta^{i}} \mid \frac{\partial h}{\partial \theta^{j}}\right),
$$

where $\theta^{i}$ are parameters of the waveform and the derivative will be computed numerically. The inner product between the two waveforms $(h \mid g)$ is defined as

$$
(h \mid g)=4 \Re \int \frac{\tilde{h}^{*}(f) \tilde{g}(f)}{S_{n}(f)} d f,
$$

with the superscript asterisk standing for complex conjugation and $S_{n}(f)$ the noise spectral density of the detector. By inverting the Fisher information matrix, we obtain the projected constraints on $\theta^{i}$ as

$$
\Delta \theta^{i}=\sqrt{\left(\Gamma^{-1}\right)_{i i}}
$$

and the covariance between $\theta^{i}$ and $\theta^{j}$ is characterized by the off-diagonal term $\left(\Gamma^{-1}\right)_{i j}$. In this paper, we will focus mainly on the CE, whose $S_{n}(f)$ is shown in Fig. 9 [107].

To see the loudness of the BNS signals, we choose a $(1.4,1.35) M_{\odot}$ BNS system as an example and plot the SNR of the pre- and postresonance signals as functions of

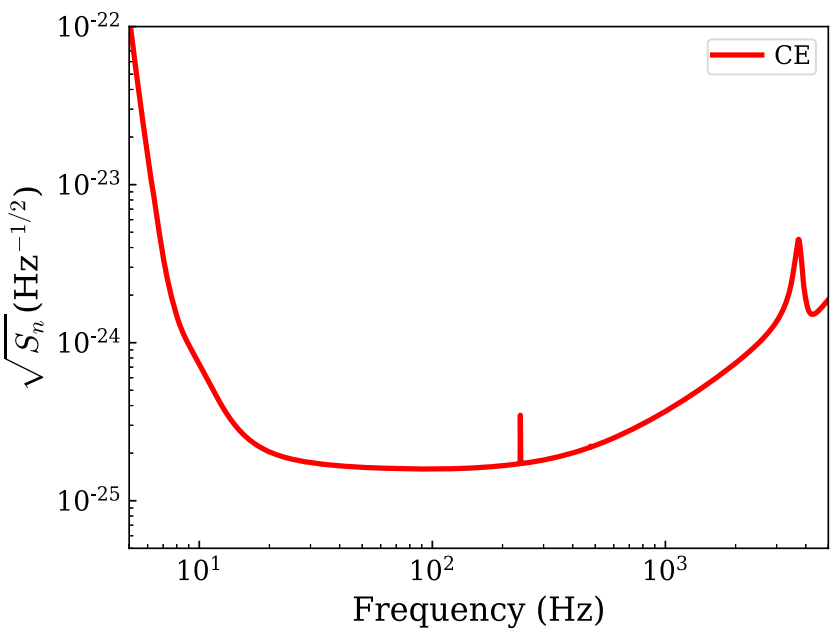

FIG. 9. The noise spectral density of the CE.

the spin frequency in Fig. 10. The system is assumed to be $100 \mathrm{Mpc}$ away and optimally oriented. Unless stated otherwise, the lower limit of our GW frequency band is $2 F_{0}=9 \mathrm{~Hz}$. We note that the CE is also sensitive to the frequency below $9 \mathrm{~Hz}$, yet it is computationally expensive to simulate the low-frequency evolution. We have checked to ensure that neglecting those signals does not lead to a significant change of parameter estimation if $\Omega_{s} \gtrsim 2 \pi \times$ $10 \mathrm{~Hz}$ (see Fig. 12). On the other hand, low-frequency signals are important if $\Omega_{s}<3 / 4 F_{0}=3.38 \mathrm{~Hz}$. Nonetheless, as we will show shortly, the $r$-mode sector does not provide such strong constraints in that regime; hence that part of parameter space is not of interest to us.

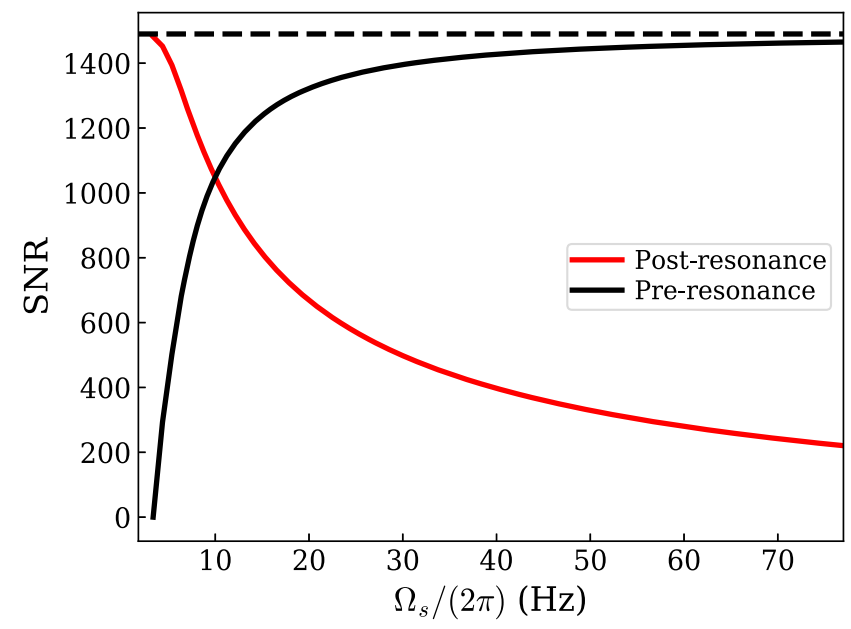

FIG. 10. SNRs of pre- and postresonance GW signals as functions of spin frequency. The BNS system is $(1.4,1.35) M_{\odot}$, optimally oriented at $100 \mathrm{Mpc}$. The EOS is GM1. As a comparison, the horizontal dash line is the SNR of the entire in-band signals. There are no preresonance signals when $3 / 4 F_{0}=3.38 \mathrm{~Hz}$ because our frequency band starts there. Recalling that the resonant orbital frequency is proportional to the spin frequency, we find that the SNR of the preresonance signal increases with the spin frequency. 
TABLE V. A summary of properties of NSs for GM1 and FPS.

\begin{tabular}{lccccc}
\hline \hline EOS & $m / M_{\odot}$ & $R / \mathrm{km}$ & \multicolumn{1}{c}{$k_{2}$} & $\bar{I}^{r}$ & $\bar{I}$ \\
\hline GM1 & 1.4 & 13.79 & 0.116 & 6.151 & 15.85 \\
GM1 & 1.35 & 13.78 & 0.121 & 6.578 & 16.87 \\
FPS & 1.4 & 10.90 & 0.0668 & 3.709 & 10.09 \\
FPS & 1.35 & 10.95 & 0.0711 & 4.000 & 10.77 \\
\hline \hline
\end{tabular}

Because the resonant orbital frequency is proportional to the spin frequency, the SNR of the preresonance signals increases with the spin frequency, and there are no preresonance signals when the spin frequency is below $3 / 4 F_{0}=3.38 \mathrm{~Hz}$. For $10 \mathrm{~Hz} \lesssim \Omega_{s} \lesssim 80 \mathrm{~Hz}$, both the preand postresonance signals are loud enough to be detected. Thus phase and time shift induced by the $r$-mode resonance (Sec. VI B) can be extracted from the waveform and can be used for parameter estimation. This is the foundation for our later discussions.

In the rest of this section, we use the same BNS system to explore the effect of $r$-mode resonance on parameter estimation. Because of the sky location, neither the inclination between the orbital angular momentum and the line of sight nor the polarization angle is of interest to us. Below we simply fix their values and consider a Fisher matrix involving only the intrinsic parameters. We mainly consider three situations. In Sec. VII B, we first investigate the case where resonances take place in both NSs. The $r$-mode DTs are treated as an independent degree of freedom (i.e., the universal relations between NS properties are not used). Then, in Sec. VIIC, we discuss the improvements on parameter estimation by incorporating the universal

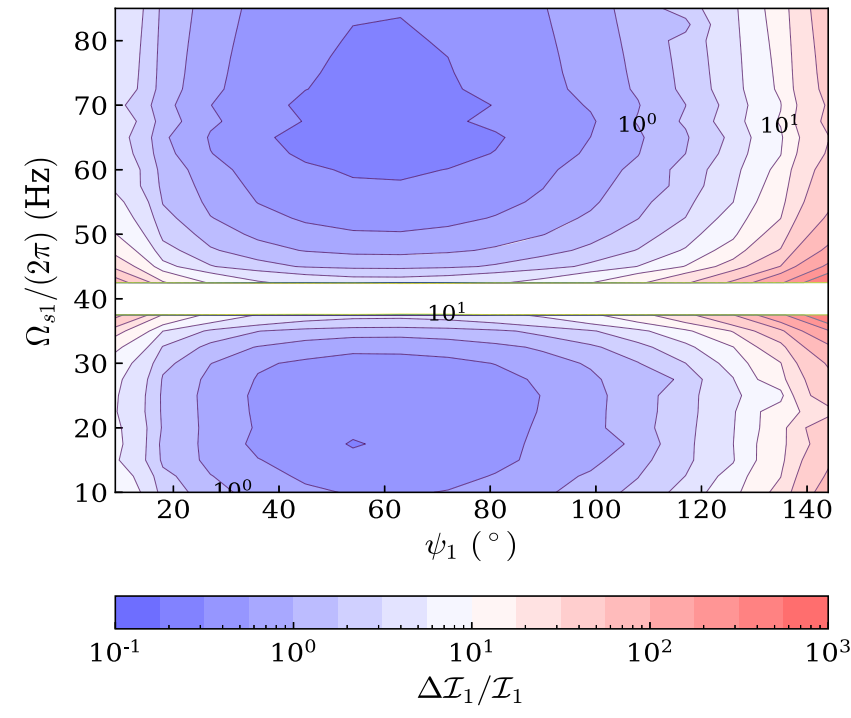

relations. Finally, in Sec. VII D, we study BHNS systems. For comparison, we consider two EOSs, GM1 and FPS, as they give, respectively, the largest and smallest radii for the same mass (see Fig. 6). The NS properties for these two EOSs are summarized in Table V. Because the features for the two EOSs are similar, we discuss mainly GM1 in the main text and put the results of FPS in Appendixes E and F.

\section{B. Case I: Two resonant NSs without the universal relations}

As we discussed in Sec. II A, for a BNS system where $r$-mode excitation takes place in each NS, $\Omega_{s i}$ and $\mathcal{I}_{i}$ can be constrained by GW. Since $r$-mode DT adds independent contributions to the GW phase evolution, simply adding this effect does not improve the existing measurement accuracy provided by other PN effects. For example, individual tidal Love numbers, as well as individual dimensionless spins, are still degenerate. Therefore, in this subsection, we focus mainly on the measurement of the $r$-mode sector itself and study the estimation accuracy for $\Omega_{s i}$ and $\mathcal{I}_{i}$. The full waveform depends on 13 parameters, including nine PP parameters, $\mathcal{M}, \Xi, \chi_{s(a)}^{(z)}, \bar{\lambda}_{f}^{s(a)}, D_{L}, t_{c}, \phi_{c}$, and four $r$-mode parameters, $\Omega_{s i}$ and $\mathcal{I}_{i}$. The Fisher matrix is 13 dimensional. We note again that here we use the normalized Love number [Eq. (31)] instead of the Love number listed in Table II.

To be concrete, let us fix the spin vector of $m_{2}, \Omega_{s 2}=$ $40 \mathrm{~Hz}$ and $\psi_{2}=7 \pi / 18$ and explore the following parameter space: $\Omega_{s 1} \in[10,85] \mathrm{Hz}, \psi_{1} \in\left[\frac{1}{20} \pi, \frac{17}{20} \pi\right]$. In Fig. 11, we show the relative errors of $\mathcal{I}_{1}$ and $\Omega_{s 1}$ as functions of

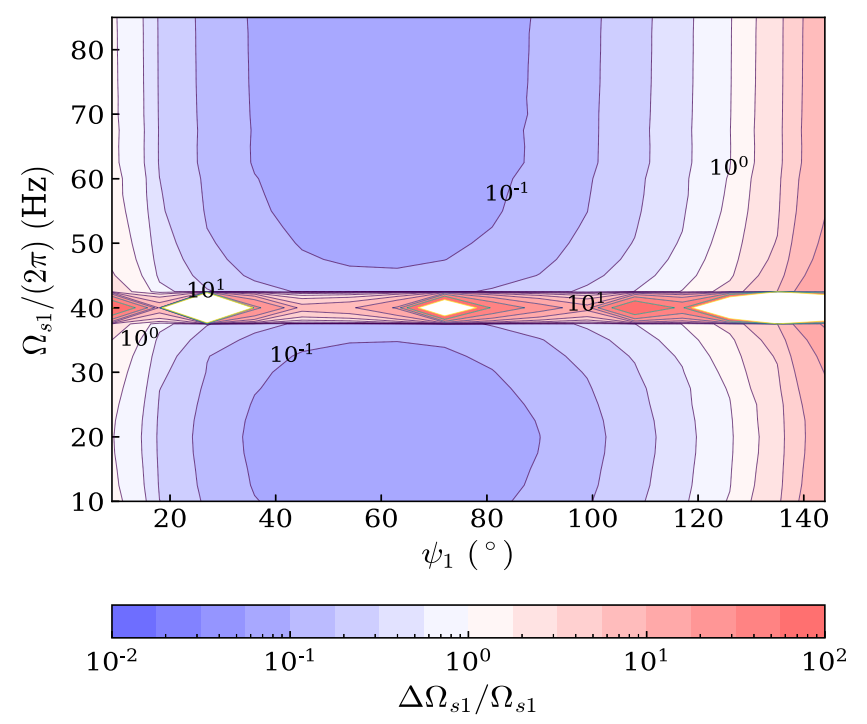

FIG. 11. Case I. Relative errors of (left panel) $\mathcal{I}_{1}$ and (right panel) $\Omega_{s 1}$ as functions of $\psi_{1}$ and $\Omega_{s 1}$, i.e., the spin configuration of $m_{1}$. The error bar is in log scale. For $m_{2}$, we fix its spin configuration as $\Omega_{s 2}=40 \mathrm{~Hz}, \psi_{2}=7 \pi / 18$. The EOS is GM1. Equation (69) shows that both $\Delta \mathcal{I}_{1} / \mathcal{I}_{1}$ and $\Delta \Omega_{s 1} / \Omega_{s 1}$ have $\psi_{1}$ dependence: $\sin ^{-2} \psi_{1} \cos ^{-4} \psi_{1} / 2$. Therefore, they diverge to infinite as $\psi_{1} \rightarrow 0, \pi$ and become the best when $\psi=\pi / 3$. In the best scenario, the constraint on $\Omega_{s 1}$ is around $6 \%$, and the one on $\mathcal{I}_{1}$ is $21.7 \%$. $\mathcal{I}_{1}$ is less constrained because $\partial h / \partial \mathcal{I}_{1}$ is suppressed by the factor $\left(1-f / f_{r}\right)$ as $f \sim f_{r}$ [Eq. (65)]. Constraints are bad when $\Omega_{s 1} \sim \Omega_{s 2}=40 \mathrm{~Hz}$ because two resonances are degenerated in $\mathrm{GW}$. 
$\Omega_{s 1}$ and $\psi_{1}$. We first observe that the relative errors of $\mathcal{I}_{1}$ and $\Omega_{s 1}$ depend on $\psi_{1}$ in a similar way; both become worse as $\psi_{1} \rightarrow 0$ and $\pi$. However, their behaviors have different causes. Recall the discussion in Sec. VIB, $\Delta \mathcal{I}_{1}$ is independent of $\mathcal{I}_{1}$, while $\Delta \Omega_{s 1} \propto \mathcal{I}_{1}^{-1}$; hence we can obtain

$$
\frac{\Delta \mathcal{I}_{1}}{\mathcal{I}_{1}} \sim \frac{\Delta \Omega_{s 1}}{\Omega_{s 1}} \sim \mathcal{I}_{1}^{-1} \sim\left(\bar{I}_{i}^{r}\right)^{-2} \sin ^{-2} \psi_{1} \cos ^{-4} \psi_{1} / 2,
$$

where Eq. (30) is used. We have checked to ensure that the dependence above is consistent with our numerical calculation. With this knowledge, we can know that constraints become the best when $\psi_{1}=\pi / 3$, where $\mathcal{I}_{1}$ is maximal. In the best case, the constraint on $\Omega_{s 1}$ is around $6 \%$, and on $\mathcal{I}_{1}$ it is around $22 \%$. Meanwhile, the measurement error $\Delta \mathcal{I}_{1} / \mathcal{I}_{1}$ is less than 1 when $\psi_{1} \in\left[18^{\circ}, 110^{\circ}\right]$; therefore, there is a large range of parameter space where one can extract meaningful constraints from GW.

Second, constraints on both $\Omega_{s i}$ and $\mathcal{I}_{i}$ become worse when $\Omega_{s 1} \sim \Omega_{s 2}=40 \mathrm{~Hz}$, because the two resonances take place at similar locations and are therefore indistinguishable from each other. In this regime, Fisher-matrix-based analysis becomes invalid, and a more detailed Bayesian analysis will be required. In the regime, the two resonances do not interfere anymore. We have checked to see that constraints on $\Omega_{s 2}$ and $\mathcal{I}_{2}$ are insensitive to the values of $\psi_{1}$ and $\Omega_{s 1}$ (except when $\Omega_{s 1} \sim \Omega_{s 2}$ ). In the best-case scenario, $\Delta \Omega_{s 2} / \Omega_{s 2}$ is around $5 \%$ and $\Delta \mathcal{I}_{2} / \mathcal{I}_{2}$ around $17 \%$.

Third, constraints on $\Omega_{s}$ are better than those on $\mathcal{I}$, because $\partial \tilde{h}^{N+r} / \partial \mathcal{I}$ is suppressed by the factor $\left(1-f / f_{r}\right)$ as $f \sim f_{r}$ [Eq. (65)]. Recalling that the spin frequency determines where the resonance takes place (in time or frequency domain), while $\mathcal{I}$ characterizes the phase shift (strength of the DT); therefore, we can conclude that GW signals are more sensitive to the location of resonance than the strength of the resonance.

Finally, we investigate the effect of EOSs. We compute the ratio of relative errors with FPS to those with GM1: $\left[\Delta \Omega_{s i}^{(\mathrm{FPS})} / \Omega_{s i}^{(\mathrm{FPS})}\right] /\left[\Delta \Omega_{s i}^{(\mathrm{GM} 1)} / \Omega_{s i}^{(\mathrm{GM} 1)}\right] \quad$ and $\left[\Delta \mathcal{I}_{i}^{(\mathrm{FPS})} / \mathcal{I}_{i}^{(\mathrm{FPS})}\right] /\left[\Delta \mathcal{I}_{i}^{(\mathrm{GM} 1)} / \mathcal{I}_{i}^{(\mathrm{GM} 1)}\right]$. In Table VI, we provide the median values of these ratios over the parameter space that we have explored. The results for different parameters are similar: those for GM1 are better than those for FPS by a factor of 2.6-3.2. The numbers are also close to the ratio of $\left(\bar{I}^{r}\right)^{-2}$, recalling that the relative errors are proportional to $\mathcal{I}^{-1} \sim\left(\bar{I}^{r}\right)^{-2}$. We can then conclude that constraints are more stringent for less compact NSs, i.e., those with harder EOSs, with an error inversely proportional to $r$-mode overlap. Detailed results for FPS are shown in Appendix E. Since the two EOSs are representative for hard and soft EOSs, the fractional error on the spin for other EOSs can be between $6 \%$ and $16 \%$. As a result, the $r$-mode resonance provides an important channel to put constraints on the spin frequency.
TABLE VI. The comparison between constraints for $\Omega_{s i}$ and $\mathcal{I}_{i}$ with two EOSs: FPS and GM1. We explore the parameter space $\Omega_{s 1} \in[10,85] \mathrm{Hz}, \psi_{1} \in\left[\frac{\pi}{20}, \frac{17}{20} \pi\right]$, while we choose $\Omega_{s 2}=40 \mathrm{~Hz}$ and $\psi_{2}=7 \pi / 18$. The median values of the ratio between two EOSs for several parameters are shown in the first four columns. Equation (69) shows that the constraint is proportional to $\left(\bar{I}_{i}^{r}\right)^{-2}$, so we also show their ratios in the last two columns for comparison. All numbers are close.

\begin{tabular}{lcccccc}
\hline \hline Parameters & $\Delta \Omega_{s 1}$ & $\Delta \Omega_{s 2}$ & $\Delta \mathcal{I}_{1}$ & $\Delta \mathcal{I}_{2}$ & $\left(\bar{I}_{1}^{r}\right)^{-2}$ & $\left(\bar{I}_{2}^{r}\right)^{-2}$ \\
\hline FPS/GM1 & 2.67 & 2.65 & 3.23 & 2.78 & 2.77 & 2.72 \\
\hline \hline
\end{tabular}

If $\Omega_{s 1}$ is further decreased to below $3.38 \mathrm{~Hz}$, we need to lower the value of $F_{0}$ to include enough preresonance signals. ${ }^{8}$ In Fig. 12, we compare the $\Delta \mathcal{I}_{1} / \mathcal{I}_{1}-\Omega_{s 1}$ and $\Delta \Omega_{s 1} / \Omega_{s 1}-\Omega_{s 1}$ relations to different values of $F_{0}$. We can see that the value of $F_{0}$ affects the constraint mildly if $\Omega_{s}>10 \mathrm{~Hz}$. Furthermore, the fractional error of $\mathcal{I}_{1}$ exceeds $100 \%$ when $\Omega_{s 1} \lesssim 6 \mathrm{~Hz}$. In this parameter regime, the $r$-mode cannot be unambiguously detected. Therefore, we focus here on $\Omega_{s}>10 \mathrm{~Hz}$, and it is enough to choose $F_{0}=4.5 \mathrm{~Hz}$ for a general calculation.

\section{Case II: Two resonant NSs + universal relations}

In the previous subsection, we studied only the estimation of parameters in the $r$-mode sector because this sector is independent of other PN effects. However, if we take into account the universal relations between NS properties, the $r$-mode sector will behave like a bridge that connects tidal and spin parameters. We will then have enough degrees of freedom in the waveform to constrain all parameters, as summarized in Table II.

\section{Neutron-star parameters}

To begin with, we need to express $\chi_{s(a)}^{(z)}$ in terms of the spin frequency and the (normalized) moment of inertia [Sec. V B]:

$$
\chi_{s(a)}^{(z)}=\frac{1}{2}\left(\bar{I}_{1} \Omega_{s 1} m_{1} \cos \psi_{1} \pm \bar{I}_{2} \Omega_{s 2} m_{2} \cos \psi_{2}\right) .
$$

We will then have 12 intrinsic parameters, including chirp mass $\mathcal{M}$, mass ratio $\Xi$, (anti)symmetric normalized Love numbers $\bar{\lambda}_{f}^{s(a)}$, two inclination angles $\psi_{i}$, two spin frequencies $\Omega_{s i}$, two $r$-mode coupling coefficients $\mathcal{I}_{i}$, and two normalized momenta of inertia $\bar{I}_{i}$. Meanwhile, we have eight constraints from GW

$$
\mathcal{M}, \quad \Xi, \quad \chi^{\mathrm{eff}}, \quad \lambda_{f}^{\mathrm{eff}}, \quad \Delta \Omega_{s i}, \quad \Delta \mathcal{I}_{i},
$$

\footnotetext{
${ }^{8}$ Constraints on $\Omega_{s 2}$ and $\mathcal{I}_{2}$ are unaffected because the two resonances are independent of each other.
} 

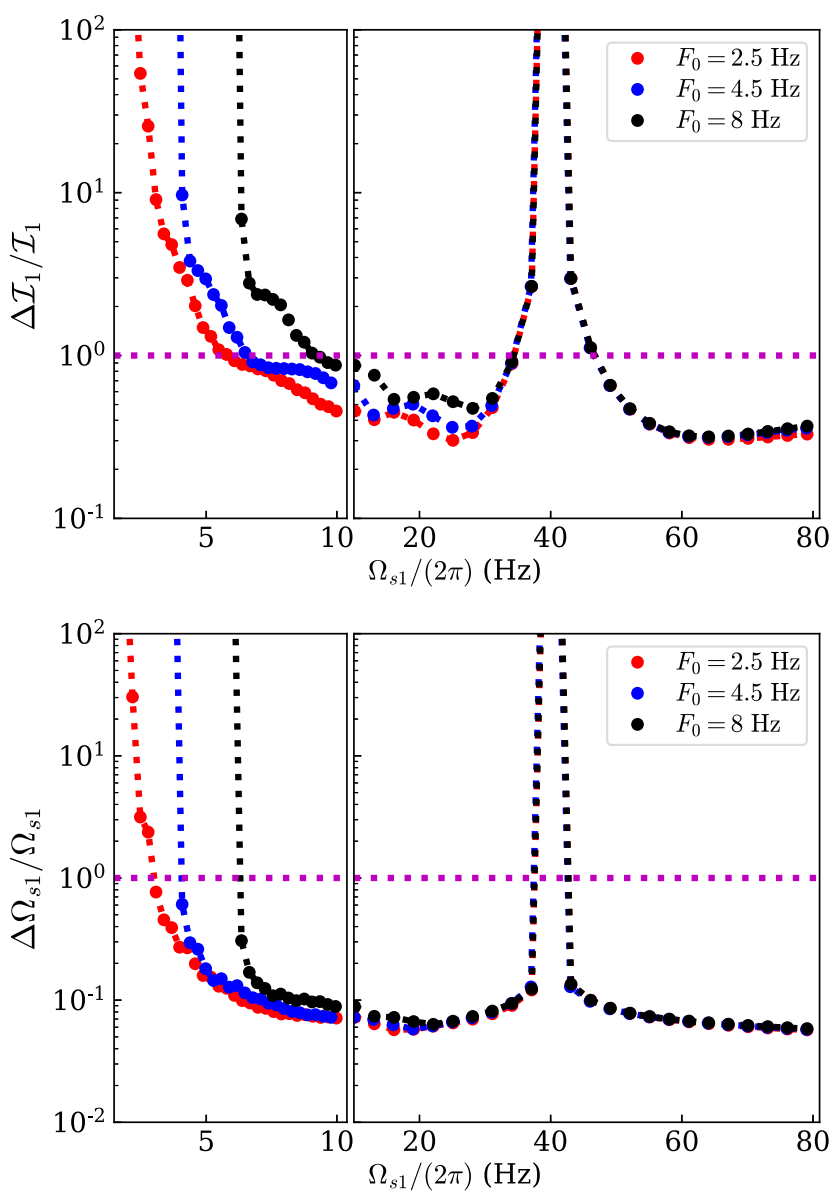

FIG. 12. Fractional errors of $\Omega_{s 1}$ and $\mathcal{I}_{1}$ as functions of $\Omega_{s 1}$, with different values of $F_{0}$. We set the spin configuration for $m_{2}$ to be the same as in Fig. 11, and $\psi_{1}=\pi / 3$. Fractional errors first decrease with $\Omega_{s 1}$, because there are more in-band preresonance signals. Then it becomes bad as $\Omega_{s 1} \sim \Omega_{s 2}$, since the two resonances are not distinguishable. The lower limit of $\Omega_{s 1} /(2 \pi)$ is taken to be $3 / 4 F_{0}$; i.e., resonance takes place initially (at orbital frequency $F_{0}$ ). We cannot get constraints on $\mathcal{I}_{1}$ and $\Omega_{s 1}$ if we further decrease the spin frequency. Those curves show that the value of $F_{0}$ affects these constraints only mildly.

and four constraints from the two universal relations

$$
\mathcal{I}_{i}=\mathcal{I}_{i}\left(\bar{\lambda}_{f}^{s(a)}, \Xi, \psi_{i}, \mathcal{M}\right), \quad \bar{I}_{i}=\bar{I}_{i}\left(\bar{\lambda}_{f}^{s(a)}\right),
$$

where we have used the definitions of $\mathcal{I}$ in Eq. (30). In principle, these 12 constraints are enough to decode the BNS system. However, as we saw in the last subsection, the constraints in Eq. (71) in practice may not be measured with extremely high statistical accuracy; the relative error on $\mathcal{I}$ can be as bad as $100 \%$. Therefore, we should be prepared for the possibility that degeneracy may not be completely broken in practice.

After using the universal relations, the Fisher matrix is 11 dimensional and corresponds to $\mathcal{M}, \Xi, \Omega_{s i}, \bar{\lambda}_{f}^{s(a)}, \psi_{i}, t_{c}$, $\phi_{c}, D_{L}$.

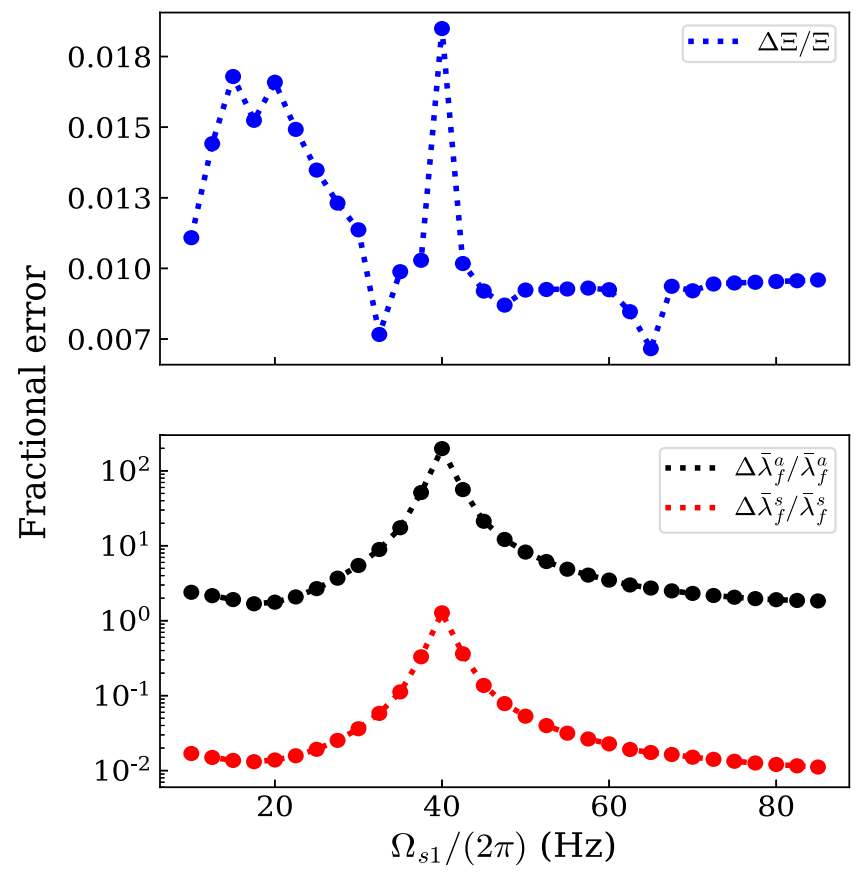

FIG. 13. Case II. Fractional errors as functions of $\Omega_{s 1}$ after incorporating universal relations. The spin configuration for $m_{2}$ is the same as in Fig. 11, and $\psi_{1}=3 \pi / 10$. The EOS is still GM1. The top panel is for $\Xi$, while the bottom panel corresponds to $\bar{\lambda}_{f}^{s(a)}$.

\section{Parameter constraints: Tidal Love numbers $\bar{\lambda}_{f}^{s, a}$}

In the lower panel of Fig. 13, we plot the dependence of $\Delta \bar{\lambda}_{f}^{s(a)} / \bar{\lambda}_{f}^{s(a)}$ on $\Omega_{s 1}$, with $\psi_{1}=3 \pi / 10, \psi_{2}=7 \pi / 18$, and $\Omega_{s 2}=2 \pi \times 40 \mathrm{~Hz}$. (We use the GM1 EOS; data for the FPS EOS are shown in Appendix F.) Constraints become worse when $\Omega_{s 1} \sim \Omega_{s 2}=2 \pi \times 40 \mathrm{~Hz}$ because two resonances take place simultaneously, making it impossible to resolve their individual features. For favorable values of $\Omega_{s 1}, \Delta \bar{\lambda}_{f}^{s}$ can be constrained as well as $\sim 1.0 \%$, while $\Delta \bar{\lambda}_{f}^{a} / \bar{\lambda}_{f}^{a}$ is $\sim 1.7$. The degeneracy between two individual tidal Love numbers is still not broken but is substantially reduced. To see this more clearly, we plot the error ellipses between $\Delta \bar{\lambda}_{f}^{a}$ and $\Delta \bar{\lambda}_{f}^{s}$ in Fig. 14 , for $\Omega_{s 1}=80 \mathrm{~Hz}$ and $\psi_{1}=\pi / 3$, when $r$ mode is either included or not included. Constraints on both directions are substantially improved by $r$-mode, but the fractional error of $\bar{\lambda}_{f}^{a}$ is still greater than 1 .

Let us note that breaking of $\bar{\lambda}_{f}^{a}$ degeneracy is difficult because the predicted values of $\bar{\lambda}_{f}^{a}$ are intrinsically very small if we make the tacit assumption that neutron stars all have the same, albeit an unknown, EOS. For example, in our case, the two neutron stars are $(1.4,1.35) M_{\odot}$, and with the GM1 EOS, $\bar{\lambda}_{f}^{a} / \bar{\lambda}_{f}^{s} \approx 0.1$. Nevertheless, it is theoretically possible that the two neutron stars do not follow the same EOS - and for this reason it is still very meaningful to dramatically reduce $\Delta \bar{\lambda}_{f}^{a}$, even if our measurement error is somewhat larger than the predicted value of $\bar{\lambda}_{f}^{a}$. 

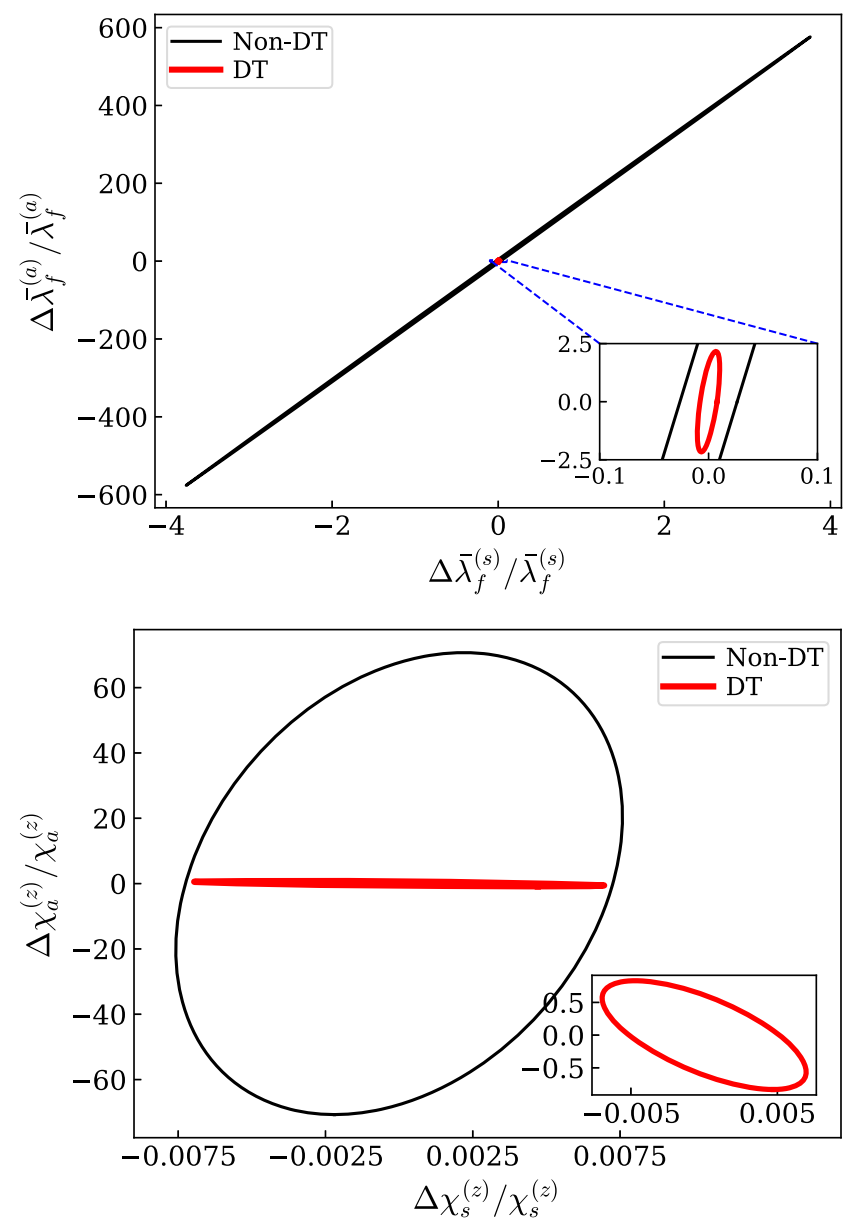

FIG. 14. The error ellipses of $\left(\Delta \bar{\lambda}_{f}^{a}, \Delta \bar{\lambda}_{f}^{s}\right)$ and $\left(\Delta \chi_{a}^{(z)}, \Delta \chi_{s}^{(z)}\right)$, with $\Omega_{s 1}=80 \mathrm{~Hz}, \Omega_{s 2}=40 \mathrm{~Hz}, \psi_{1}=\pi / 3$, and $\psi_{2}=7 \pi / 18$. The black curve is the result of PN effects (including the adiabatic tidal effect and spin-orbit coupling). The red curve is the result after including $r$-mode resonances (with universal relations). For those ellipses, both directions are improved by resonances.

As we turn to the individual Love numbers of the two neutron stars, $\bar{\lambda}_{f}^{(i)}$, we find that their errors are still correlated in general. For some special cases, the individual Love numbers can be sufficiently well constrained. In Table VII, we show one example with $\Omega_{s 1}=80 \mathrm{~Hz}$, $\psi_{1}=\pi / 3, \Omega_{s 2}=40 \mathrm{~Hz}, \psi_{1}=7 \pi / 18$, and GM1 EOS. Relative errors of two individual tidal Love numbers are around $20 \%$.

We then study the improvement of constraints by comparing the results above to those of pure PN effects (i.e., the adiabatic tidal effect). The improvement factor, Imp. $\Delta \bar{\lambda}_{f}^{s(a)} / \bar{\lambda}_{f}^{s(a)}$, is defined as the ratio between constraints from two sides (the factor is larger than 1 when the $r$-mode enhances the constraint). The results are shown in Table VIII. We can see that the constraints on $\Delta \bar{\lambda}_{f}^{s(a)} / \bar{\lambda}_{f}^{s(a)}$ are improved. In the best case, the factor is around 300-400.
TABLE VII. A special case where the individual normalized Love numbers and inclination angles are well constrained. Two NSs have spin $\Omega_{s 2}=40 \mathrm{~Hz}, \psi_{2}=7 \pi / 18, \Omega_{s 1}=80 \mathrm{~Hz}$, and $\psi_{1}=\pi / 3$. The EOS is GM1.

\begin{tabular}{ccccc}
\hline \hline Parameters & $\Delta \bar{\lambda}_{f}^{(1)} / \bar{\lambda}_{f}^{(1)}$ & $\Delta \bar{\lambda}_{f}^{(2)} / \bar{\lambda}_{f}^{(2)}$ & $\Delta \psi^{(1)}(\mathrm{rad})$ & $\Delta \psi^{(2)}(\mathrm{rad})$ \\
\hline Constraints & 0.26 & 0.22 & 0.18 & 0.28 \\
\hline \hline
\end{tabular}

TABLE VIII. Summary of the effect of including $r$-mode resonance in parameter constraints. The second column gives the best fractional errors for $\Xi$ and $\bar{\lambda}_{f}^{s(a)}$ achievable when we vary $\psi_{1}$ and $\Omega_{s 1}$. These fractional errors are generally improved relative to those achievable including only $\mathrm{PN}$ effects. In the third and forth columns, we list the best and worst improvement factors for each parameter, as we vary $\psi_{1}$ and $\Omega_{s 1}$.

\begin{tabular}{lccc}
\hline \hline Parameters & $\begin{array}{c}\text { Best } \\
\text { constraints }\end{array}$ & $\begin{array}{c}\text { Best } \\
\text { improvement }\end{array}$ & $\begin{array}{c}\text { Worst } \\
\text { improvement }\end{array}$ \\
\hline$\Delta \bar{\lambda}_{f}^{s} / \bar{\lambda}_{f}^{s}$ & $10^{-2}$ & 389 & 1 \\
$\Delta \bar{\lambda}_{f}^{a} / \bar{\lambda}_{f}^{a}$ & 1.84 & 336 & 1 \\
$\Delta \Xi / \Xi$ & $5 \times 10^{-3}$ & 11.6 & 1 \\
\hline \hline
\end{tabular}

\section{Parameter constraints: Mass ratio $\Xi$ and spins $\chi_{a, s}^{(z)}$}

The measurement of $\Delta \chi_{a}^{(z)}$ and $\Delta \chi_{s}^{(z)}$ can also benefit from universal relations. ${ }^{9}$ As shown in the lower panel of Fig. 14, while the improvement in $\chi_{s}^{(z)}$ is mild, the constraint on $\chi_{a}^{(z)}$ is improved by a factor of $\sim 120$. Therefore, the degeneracy between individual dimensionless spin is also reduced.

The case for $\Xi$ is similar. Its correlation with other parameters is reduced by the $r$-mode DT and the universal relations. Compared to constraints from pure PN effects, the error in $\Xi$ can be improved by a factor of $1-11.6$, as summarized in Table VIII. In the first row of Fig. 13, we also present its fractional error as a function of $\Omega_{s 1}$. We can see that $\Delta \Xi / \Xi$ is insensitive to $\Omega_{s}$. Generally, it can be constrained to $\sim 1.3 \%$.

It is also well known that the estimation errors of $\Xi, \bar{\lambda}_{f}^{s}$, and $\chi_{s}^{(z)}$ are correlated in the absence of $r$-mode resonance. The effects of incorporating $r$-mode resonances are shown in Fig. 15. Whereas in Refs. [1,4] the error reduction relies on imposing an observational-based prior on $\chi_{z}$, once the $r$-mode resonances are taken into account, the mutual correlations between parameters are significantly reduced. As suggested in Ref. [76], the $r$-mode DT indeed dramatically improves the inference accuracy on both the tidal deformability and the NS component masses. The latter can

\footnotetext{
${ }^{9}$ These are not independent variables in this subsection. Their constraints are obtained by error propagation.
} 

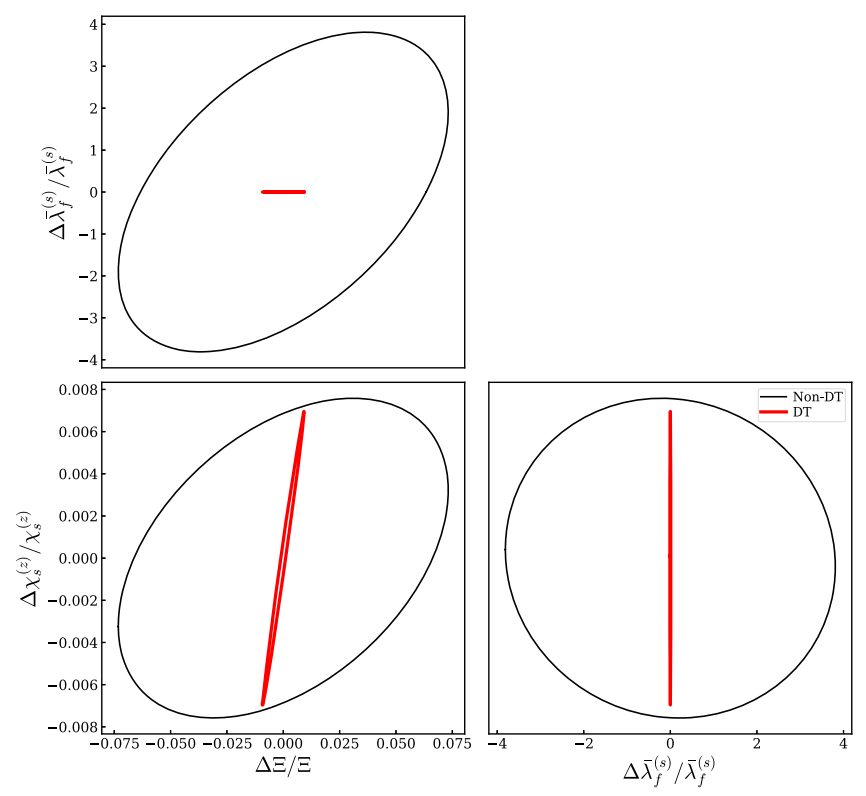

FIG. 15. Same as Fig. 14. The error ellipses between $\Xi-\bar{\lambda}_{f}^{s}-\chi_{s}^{(z)}$.

be further converted into an indication of the maximum mass of NSs with a population of events. We thus conclude that the $r$-mode DT plays a crucial role in constraining the nature of NS EOSs.

\section{Parameter constraints: Inclination angles}

In addition to improving constraints, universal relations also provide the information of inclination angles $\psi_{i}$, which is hard to access through other PN effects. In Fig. 16, we show $\Delta \psi_{i}$ as functions of $\Omega_{s 1}$. Generally speaking, $\psi_{1}$ and $\psi_{2}$ are correlated. In the best case, $\Delta \psi_{1} \sim \Delta \psi_{2} \sim 0.09 \mathrm{rad}$. We note that determining this angle may play a significant role in astrophysics, as it allows the constraining of the NS natal kicks, i.e., kicks received by NSs at their formation due to asymmetric supernova explosions (see, e.g., Refs. [108-110]). This may further shed light on models and theories of core-collapse supernova [111,112].

\section{If only one of the NSs is resonant}

If one of the NSs (e.g., $m_{2}$ ) in the binary system rotates at very low frequency, its $r$-mode resonance is not in band anymore, and $r$-mode resonance does not provide enough constraints to decode the entire system. In fact, we find that the two inclination angles $\psi_{i}$ are highly correlated and totally unmeasurable. Nevertheless, the measurement of Love number can still benefit from the single, in-band resonance. As an example, we still adopt a BNS system with $(1.4,1.35) M_{\odot}$ and $\psi_{1}=\pi / 3, \Omega_{s 1}=40 \mathrm{~Hz}$. The value of $\Omega_{s 2}$ is taken to be small enough that the $r$-mode of $m_{2}$ is not excited in band. In Fig. 17, we show contours between $\bar{\lambda}_{f}^{s}$ and $\bar{\lambda}_{f}^{a}$ with or without DT. We can see that they

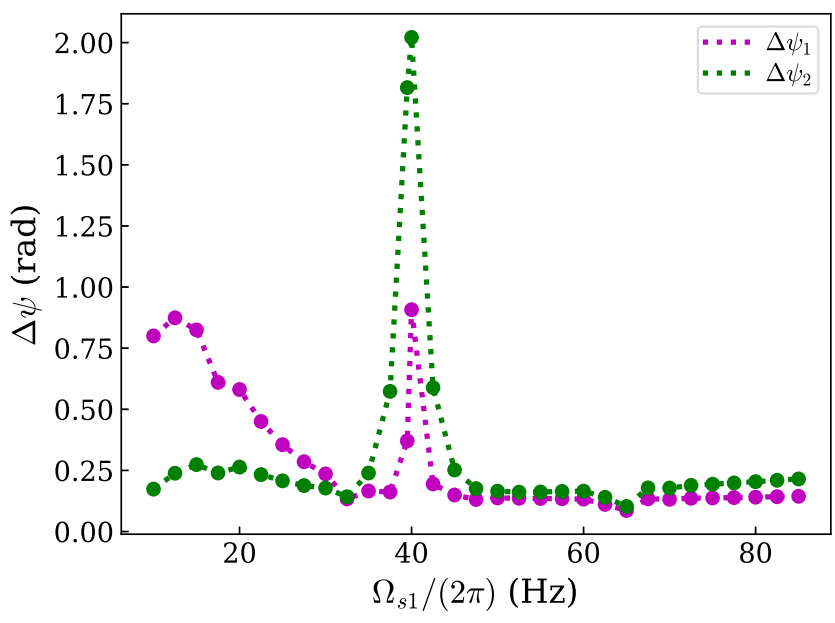

FIG. 16. Case II. Constraints on inclination angles $\psi_{i}$ as functions of $\Omega_{s 1}$. Spin configurations are the same as in Fig. 11 and $\psi_{1}=3 \pi / 10$. Generally speaking, $\psi_{1}$ and $\psi_{2}$ are correlated. In the best case, $\Delta \psi_{1} \sim \Delta \psi_{2} \sim 0.09 \mathrm{rad}$.

are similar to the case of two resonances in Fig. 14: even with only one resonance, the degeneracy between $\bar{\lambda}_{f}^{s(a)}$ can still be substantially reduced.

\section{Case III: BHNS}

For a BHNS system, the $r$-mode resonance takes place only once before merger. As shown in Table III, there are eight parameters for the system: $\mathcal{M}, \Xi, \bar{\lambda}_{f}^{(1)}, \bar{I}_{1}, \Omega_{s 1}, \mathcal{I}_{1}, \psi_{1}$, and $\chi_{2}^{(z)}$. And there are eight constraints from GWs and universal relations. Hence the degeneracies can be reduced, or even broken.

To study this case, we choose a BHNS system with $(1.4,10) M_{\odot}$. The NS is assumed to spin at $30 \mathrm{~Hz}$, and the

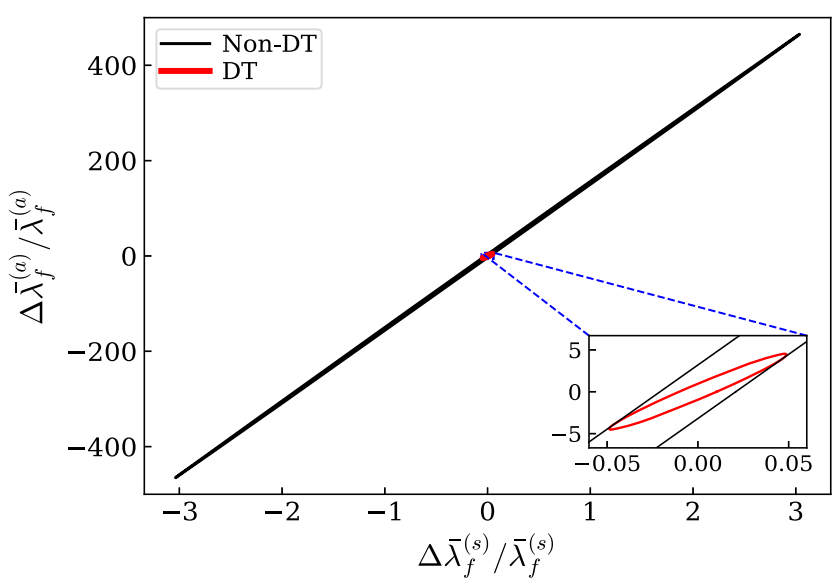

FIG. 17. Similar to the top panel of Fig. 14 but with only one inband $r$-mode resonance. For the binary system, we choose $\Omega_{s 1}=40 \mathrm{~Hz}, \psi_{1}=\pi / 3$. The value of $\Omega_{s 2}$ is taken to be small enough that the $r$-mode of $m_{2}$ is not excited in band. The degeneracy between $\bar{\lambda}_{f}^{s(a)}$ can still be reduced significantly. 

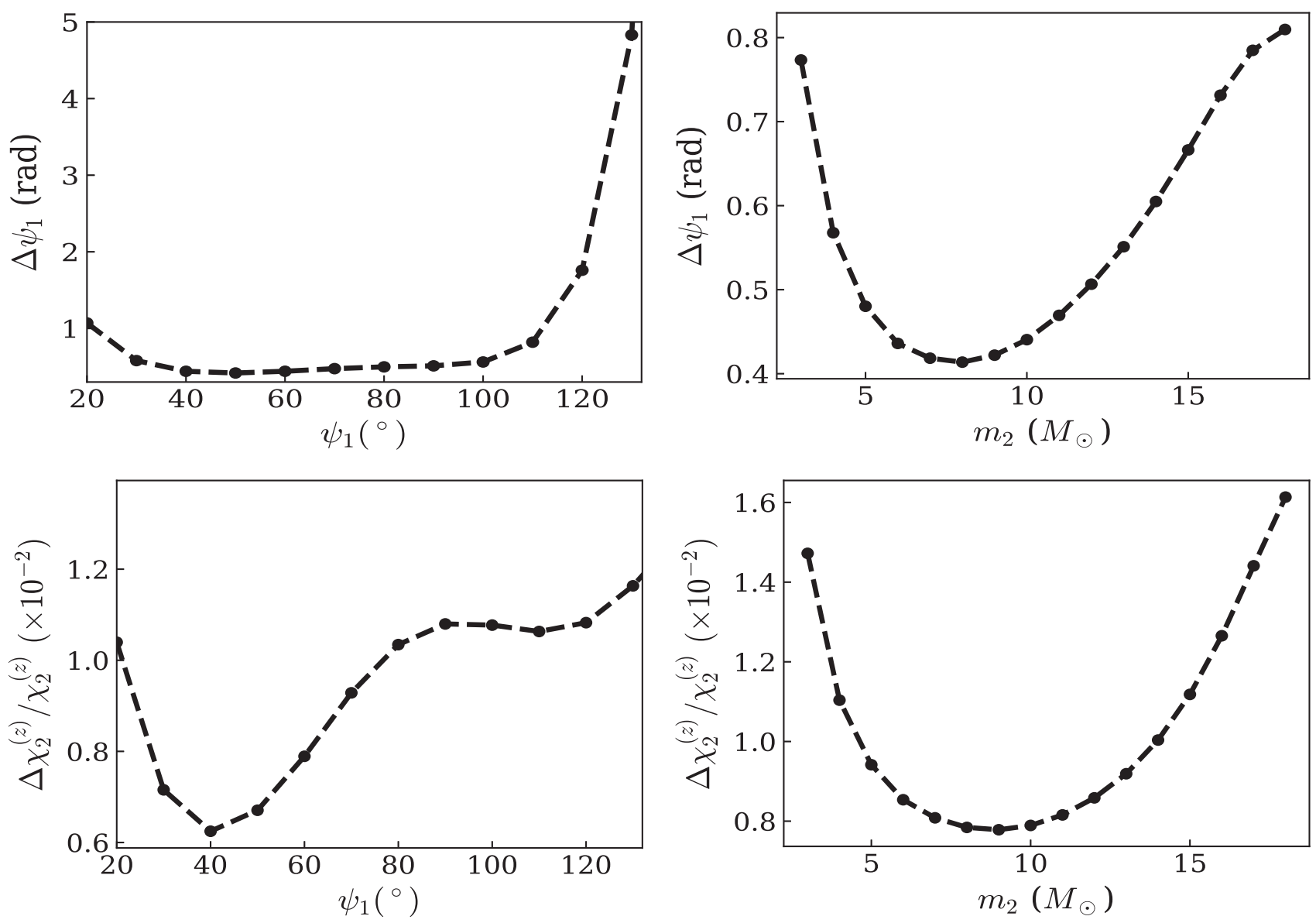

FIG. 18. Case III. Constraints on several parameters in the case of a BHNS system. We choose $\Omega_{s 1}=30 \mathrm{~Hz}$ and $\chi_{2}^{(z)}=0.1$. The EOS is GM1, and the binary system is $100 \mathrm{Mpc}$ from the detector. In the left panels, we show constraints as functions of $\psi_{1}$, where we fix the mass of the $\mathrm{BH}$ to be $10 M_{\odot}$, whereas in the right panels we study their dependence on the mass of the $\mathrm{BH} m_{2}$, with $\psi s_{1}=\pi / 3$. Using universal relations, the degeneracy of parameters is totally broken, where $\Delta \psi$ is $\sim 1$ rad and the relative errors of $\chi_{2}^{(z)}$ are $\sim 1 \%$.

EOS is GM1. As for the BH, we assume that $\chi_{2}^{(z)}=0.1$. The distance of the system is $100 \mathrm{Mpc}$. The results are shown in the left column of Fig. 18. In this case, the degeneracy between the two individual spins is completely broken. For $\chi_{2}^{(z)}$, it is constrained to $\sim 1 \%$, and $\psi_{1}$ is constrained to $\sim 1 \mathrm{rad}$.

We further investigate the effect of $\mathrm{BH}$ mass on constraints by varying $m_{2}$ while fixing $\Omega_{s 1}=30 \mathrm{~Hz}$ and $\psi_{1}=\pi / 3$. The distance in the system is still $100 \mathrm{Mpc}$. In the right column of Fig. 18, we show the constraints as functions of the $\mathrm{BH}$ mass. We can see that both constraints first decrease with $m_{2}$, because of the increase of SNR. If we further increase $m_{2}$, postresonance signals then will be reduced, and the constraints will become worse accordingly.

\section{CONCLUSION}

In this paper, we studied the tidal excitation of $r$-mode by the gravitomagnetic force in coalescencing NS binaries. We began with a brief review on the dynamics of these systems: the $r$-mode is excited by the gravitomagnetic field from the companion, while the induced current quadrupole moment gives rise to an acceleration back to the orbit. By assuming the orbit to be quasicircular, we obtained a coupled EOM. Next we numerically integrated the coupled set of EOMs and discussed features of solutions. We confirmed that the pre- and postresonance orbital evolution can be well described by two PP orbits, as proposed in FR07 [72]. The postresonance PP orbit is related to the preresonance one through a jump in orbital frequency at the resonance. We subsequently investigated the tidal evolution by extending Ref. [53] to the $r$-mode and providing analytic formulas for tidal evolution that are accurate in all regimes: adiabatic, resonance, and postresonance. Separately, using the TOV equation, we found a universal relation between the normalized $r$-mode overlap $\bar{I}^{r}$ of a neutron star and its normalized tidal Love number $\bar{\lambda}_{f}$.

We then moved on to the wave zone and studied gravitational waves emitted by such binaries. We constructed a hybrid GW waveform that combines several SPA models with results from numerical integrations of the coupled EOM. This waveform contains information from 
the $r$-mode resonance, the adiabatic tidal effect, and spinorbit coupling. To understand the effect of the $r$-mode DT on GW waveforms, we adopted the model in FR07: the $r$-mode induces phase and time shifts in GW. We found this model to be quantitatively accurate. Finally, with the hybrid waveform, we calculated the Fisher information matrix to investigate how $r$-mode resonances improves the parameter estimation accuracy. We studied mainly three cases: binary NS systems with $r$-mode resonances in NSs, binary NS systems including $r$-mode resonances together with universal relations between NS properties, and BHNS binary systems.

We had a variety of interesting results. First, the excitation of the $r$-mode is described mainly by two parameters: spin frequency $\Omega_{s}$ and $r$-mode coupling coefficient $\mathcal{I}$. The spin frequency $\Omega_{s}$ determines when the resonance takes place during the inspiral, and $\mathcal{I}$ determines the phase and time shifts induced by the $r$-mode resonance. Choosing a $(1.4,1.35) M_{\odot}$ BNS system at $100 \mathrm{Mpc}$ away as example, we found that when the inclination angle is within the range of $\left[18^{\circ}, 110^{\circ}\right]$ (and if $\Omega_{s 1,2}$ are not too close), the measurement errors of $\mathcal{I}_{i}$ and $\Omega_{s i}$ are less than $100 \%$, where the Fisher analysis is valid and we can extract meaningful information from GW. The best constraint on $\Omega_{s}$ is around $6 \%$ with $3 \mathrm{G}$ detectors, whereas for $\mathcal{I}$ the value is around $22 \%$. The constraint on $\mathcal{I}$ is worse than $\Omega_{s}$ because $\partial \tilde{h}^{N+r} / \partial \mathcal{I}$ is suppressed by the factor $\left(1-f / f_{r}\right)$ as $f \sim f_{r}$ [Eq. (65)]. In other words, the waveform is more sensitive to the location of the resonance than to the phase shift. With the analytic model for the $r$-mode, we found $\Delta \mathcal{I} / \mathcal{I} \sim \Delta \Omega_{s} / \Omega_{s} \sim$ $\sin ^{-2} \psi \cos ^{-4} \psi / 2$ [Eq. (69)]. This is consistent with our numerical calculations. The formula shows that the constraint is best when $\psi=\pi / 3$, while there is no constraint as $\psi \rightarrow 0, \pi$. We also found that two resonances in each star do not get strongly correlated except for $\Omega_{s 1} \sim \Omega_{s 2}$, when effects from the two resonances become indistinguishable.

Second, with the help of the universal relations, the $r$-mode resonance behaves like a bridge that connects the adiabatic tidal effect and the spin-orbit coupling. In principle, for systems which have two $r$-mode resonances, we can obtain as many constraints as free parameters in GW. This situation is contrary to the case without DT, where the universal relation requires additional parameters to be incorporated. This is because if one wants to use the I-Love relation to connect the adiabatic tidal effect and the spin-orbit coupling, four more free parameters (inclination angle $\psi_{i}$ and spin frequency $\Omega_{s i}$ ) should be introduced. In the absence of $r$-mode resonance, they cannot be constrained at all.

Although the $r$-mode resonance provides enough constraints to decode the BNS system, some constraints are not very accurate in practice. For example, errors on $\mathcal{I}_{i}$ sometimes are as large as $100 \%$, where the information is not meaningful. This will diminish the role of the $r$-mode in degeneracy breaking. Nevertheless, our calculations show that two individual normalized Love numbers are still significantly correlated in most cases. The best relative errors of symmetric normalized Love number is $\sim 1.3 \%$, while it is 1.84 for the antisymmetric normalized Love number. Both of them are improved by factors of $~ 300-400$ in the best-case scenario relative to those that come solely from PN effects. In favorable cases, the normalized Love numbers of individual NS can be sufficiently well constrained. As shown in Table VII, each normalized Love number is constrained to $20 \%$, which significantly improves our understanding of the NS EOS. Meanwhile, the $r$-mode DT allows us to put constraints on the inclination angle between the spin and orbital angular momentum, which is hard access through other PN effects. In the best-case scenario, each inclination angle is constrained to $0.09 \mathrm{rad}$. This could potentially constrain the NS natal kick and hence the supernova explosion mechanism. The other benefit from the universal relations is constraints on the mass ratio $\Xi$, which is known to have correlated errors with $\bar{\lambda}_{f}^{s}$ and $\chi_{s}^{(z)}$ in the absence of DT. After including the $r$-mode DT and universal relations, $\Xi$ measurement can be improved by a factor of 1-11.6. For most cases, its fractional error is around $1 \%$. An improved estimation accuracy on $\Xi$ means better accuracy for the component masses. This could constrain the maximum mass of NSs with a large number of detection and shed light on the NS EOS in a way that is complementary to the information derived from tidal deformability.

Third, for BHNS systems, we can also obtain as many constraints as parameters. As a result, degeneracies are totally broken. Choosing a BHNS system for an example, we found $\Delta \psi_{1} \sim 1 \mathrm{rad}$, and $\Delta \chi_{2}^{(z)} / \chi_{2}^{(z)} \sim 1 \%-2 \%$.

Our results show that $r$-mode resonance will be an important channel for $3 \mathrm{G}$ detectors to extract information about the NSs. Since the excitation requires NSs to spin only at tens of hertz, these events are quite generic in coalescing binaries that have NSs. Therefore, to develop an accurate GW waveform from these systems seems to be necessary in the future. Our numerical calculations of $r$-mode are only on Newtonian order, and PN effects are incorporated in a naive way. Also, the corrections of DT to PN terms are not considered here. Therefore, one possible avenue for future work is to perform a systematic study on the scenario with relativity. This includes to couple the gravitomagnetic force with rotational modes of relativistic stars ${ }^{10}$ with the formalism in Ref. [66], and to study the orbital evolution with either the PN approach [83] or the EOB formalism [35,57]. As pointed out by Idrisy et al. [68], there is a more than $10 \%$ variance for the mode frequency of relativistic stars, depending on the compactness. This is on the same order of statistical accuracy of $\Omega_{s}$ in our paper. Therefore, the relativistic corrections would be important in this case. It would also be interesting to see how relativistic corrections to the $\bar{I}^{r}-\bar{\lambda}_{f}$ universal relation changes the parameter estimation.

\footnotetext{
${ }^{10}$ There is no pure $r$-mode in relativistic barotropic stars.
} 
The other direction would be numerical relativity. Although the excitation of an $f$-mode was observed in a recent study [113], the simulation of an $r$-mode is more difficult to achieve with a current version of a numerical relativity code such as SpEC [114], since the mode amplitude of an $r$-mode is much smaller. A typical Lagrangian displacement of an $r$-mode is only $10^{-4}$ the radius of a NS. It requires much more resolution to resolve an $r$-mode. However, with the upgraded version of SpEC, SpECTRE [115], this may be possible in the near future.

Furthermore, our paper focuses mainly on the $(2,2)$ $r$-mode in barotropic NSs. As pointed out by Poisson recently [73], four inertial modes can be excited by the gravitomagnetic force before merger. Meanwhile, for NSs with buoyancy, there is also the $(2,1) r$-mode, which plays a role as important as the $(2,2)$ mode [72]. These modes have different $\psi$ dependence and contain different information about NSs. Therefore, they can further reduce the degeneracy of parameters and put more constraints on the inclination angle $\psi$. On the other hand, the detection of the $(2,1)$ mode can confirm the existence of buoyancy in cold NSs, so it is worth incorporating these modes in the future.

A caveat, however, is that our analysis and the studies mentioned above all have assumed that the matter inside the NS behaves as a normal fluid. In reality, superfluidity may be expected in cold NSs [116] and may lead to richer dynamics than what we considered here thanks to its twofluid nature (see, e.g., Ref. [117]). Reference [118] showed that the superfluid $r$-modes characterized by a common flow of neutrons and protons reduce to their normal-fluid counterparts (i.e., the $r$-mode studied in our work) in the slow-rotation limit. On the other hand, Ref. [117] argued for the existence of a new class of $r$-modes whose fluid motion is such that neutrons and protons are countermoving. This new class of $r$-modes is not accounted for in this study and is deferred to future studies.

Meanwhile, we ignored damping on the $r$-mode due to microphysical processes in the NS. While viscous damping and nonlinear saturation may play a critical role for the $r$-mode instability in newly born NSs and/or x-ray binaries [119], its effect might be subdominant in coalescing NS binaries given the very short duration of tidal excitation $[<1 \mathrm{~S}$; Eq. (A9)] relative to the typical viscous damping timescale of $10^{4} \mathrm{~s}$ [118]. Nevertheless, sufficiently large uncertainty remains in our current understanding of the NS microphysics, and future explorations of the dissipation of saturation of $r$-modes under various astrophysical contexts will be of great value.

Finally, we want to emphasize that we treated the two universal relations as exact relations. However, as pointed out in Ref. [120], even the most insensitive relations still have residual variability with respect to EOSs and could lead to systematic bias in parameter estimations for $3 \mathrm{G}$ detectors potentially comparable to the statistical uncertainties. Therefore, studying the impact of such EOS variability would be another interesting direction to take.

\section{ACKNOWLEDGMENTS}

We thank the LIGO Extreme Matter working group for the useful comments during the preparation of this work. We also thank Chris van den Broeck for useful comments. The research of S. M. and Y.C. is supported by the Simons Foundation (Grant No. 568762), the Brinson Foundation, and the National Science Foundation (Grants No. PHY2011968, No. PHY-2011961, and No. PHY-1836809). H. Y. is supported by the Sherman Fairchild Foundation. The computations presented here were conducted on the Caltech High Performance Cluster, which is partially supported by a grant from the Gordon and Betty Moore Foundation.

\section{APPENDIX A: JUSTIFICATION FOR IGNORING THE PP PRECESSION}

When the spins $S_{1,2}$ are misaligned with respect to the orbital angular momentum $\boldsymbol{L}$, various precession effects will occur and, in principle, modify the dynamics when the orbit is both close to and far from a mode's resonance. We will show quantitatively that all the precession-induced corrections are small and therefore can be safely ignored.

Specifically, we have $[80,121]$

$$
\begin{gathered}
\frac{d \hat{\boldsymbol{S}}_{1}}{d t}=\boldsymbol{\Omega}_{\mathrm{dS}}^{(1)} \times \hat{\boldsymbol{S}}_{1}, \\
\frac{d \hat{\boldsymbol{S}}_{2}}{d t}=\boldsymbol{\Omega}_{\mathrm{dS}}^{(2)} \times \hat{\boldsymbol{S}}_{2}, \\
\frac{d \hat{\boldsymbol{L}}}{d t}=\left[\boldsymbol{\Omega}_{\mathrm{LT}}^{(1)}+\boldsymbol{\Omega}_{\mathrm{LT}}^{(2)}\right] \times \hat{\boldsymbol{L}},
\end{gathered}
$$

where

$$
\boldsymbol{\Omega}_{\mathrm{dS}}^{(1,2)}=\frac{3\left(m_{2,1}+\mu / 3\right)}{2 D} \boldsymbol{\Omega}_{\mathrm{orb}} \hat{\boldsymbol{L}}
$$

is the rate of the leading-order de Sitter precession of the spins induced by the orbital angular momentum $\boldsymbol{L}$ and

$$
\boldsymbol{\Omega}_{\mathrm{LT}}^{(1,2)}=\frac{S_{1,2}\left(4+3 m_{2,1} / m_{1,2}\right)}{2 D^{3}} \hat{\boldsymbol{S}}_{2}
$$

is the rate of the Lense-Thirring precession of $\boldsymbol{L}$ due to $\boldsymbol{S}_{1,2}$. The hat stands for the unit vector along the direction of the corresponding quantity.

As a result of the de Sitter precession of $S_{1}$, the resonance frequency of the $r$-mode in $m_{1}$ should be shifted by $\Omega_{\mathrm{dS}}^{(1)}$ with

$$
\frac{\Omega_{\mathrm{dS}}^{(1)}}{2 \pi} \simeq 0.8 \times\left(\frac{f_{r}}{80 \mathrm{~Hz}}\right)^{5 / 3},
$$

assuming a binary with $m_{1} \simeq m_{2} \simeq 1.4 M_{\odot}$ and evaluating at $f=f_{r}$, with $f_{r}$ the resonant frequency [Eq. (61)]. The above shift is $\sim 1 \%$ of the spin frequency, about 10 times smaller than the typical statistical error of $\sim 10 \%$ (see, e.g., Fig. 11); it can thus be neglected. 


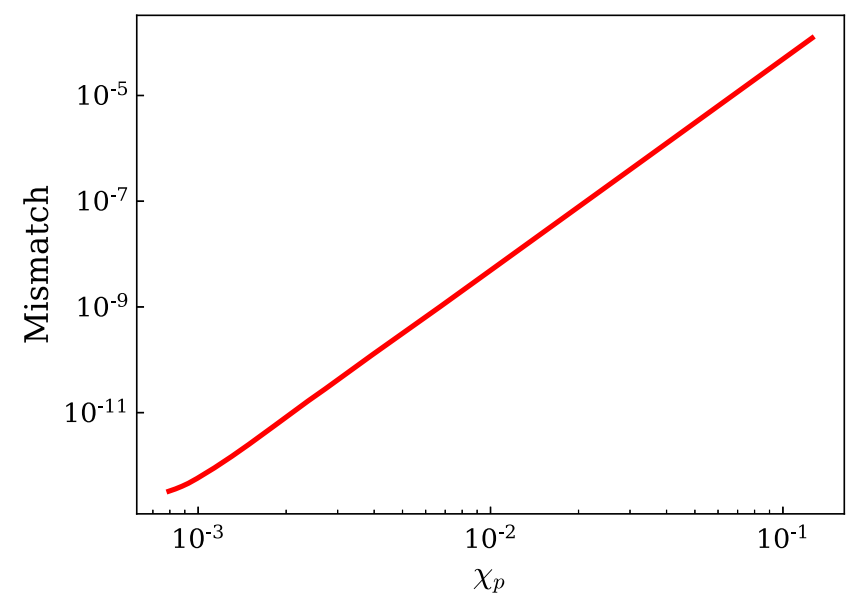

FIG. 19. Mismatch between a precessing waveform $\chi_{\mathrm{p}} \neq 0$ and a nonprecessing but otherwise identical one.

Meanwhile, the Lense-Thirring precession of the orbital angular momentum together with the spin-spin interaction will cause the inclination angle of the spin of NS, $\psi_{1(2)}$, to evolve slowly as [122]

$$
\frac{d}{d t} \cos \psi_{1}=\Omega_{\Delta \psi_{1}} \hat{\boldsymbol{S}}_{1} \cdot\left(\hat{\boldsymbol{S}}_{2} \times \hat{\boldsymbol{L}}\right),
$$

where $\Omega_{\Delta \psi_{1}}=3(1+q) S_{2} / 2 q D^{3} \simeq(6 / 7) \Omega_{\mathrm{LT}}^{(2)}$ for nearly equal-mass binaries with $q \equiv m_{2} / m_{1} \simeq 1$. We can thus define a timescale $\tau_{\Delta \psi_{1}}=1 / \Omega_{\Delta \psi_{1}}$, which is given by

$$
\tau_{\Delta \psi_{1}} \simeq 70 \mathrm{~s}\left(\frac{f_{r}}{80 \mathrm{~Hz}}\right)^{-2}\left(\frac{\chi_{2} \sin \psi_{2}}{0.02}\right)^{-1} .
$$

On the other hand, the duration of the resonance is given by [Eq. (48b)]

$$
\tau_{\mathrm{r}} \simeq\left(\frac{1}{\dot{\Omega}_{r}}\right)^{1 / 2}=0.52 \mathrm{~s}\left(\frac{f_{r}}{80 \mathrm{~Hz}}\right)^{-11 / 6} .
$$

We thus see that $\psi_{1}$ is a well-defined quantity at resonance. Moreover, it is well defined throughout the entire postresonance evolution, which lasts about $4 \mathrm{~s}$ (Fig. 4), much shorter than $\tau_{\Delta \psi_{1}}$.

The above two points allow us to conclude that the modifications due to precession are indeed negligible during mode resonances. We now consider their effects away from resonance. To do so, we drop the $r$-mode effects and use LALSuite [123] to generate PP waveforms with IMRPhenomPv2 approximation [80,124].

In Fig. 19, we compute the mismatch of a precessing waveform (parametrized in terms of $\chi_{\mathrm{p}}$ ) along with a nonprecessing but otherwise identical one. Here the mismatch is defined as

$\operatorname{Mismatch}\left(h_{1}, h_{2}\right)=1-\max _{t_{c}, \phi_{c}} \frac{\left(h_{1} \mid h_{2}\right)}{\sqrt{\left(h_{1} \mid h_{1}\right)\left(h_{2} \mid h_{2}\right)}}$.
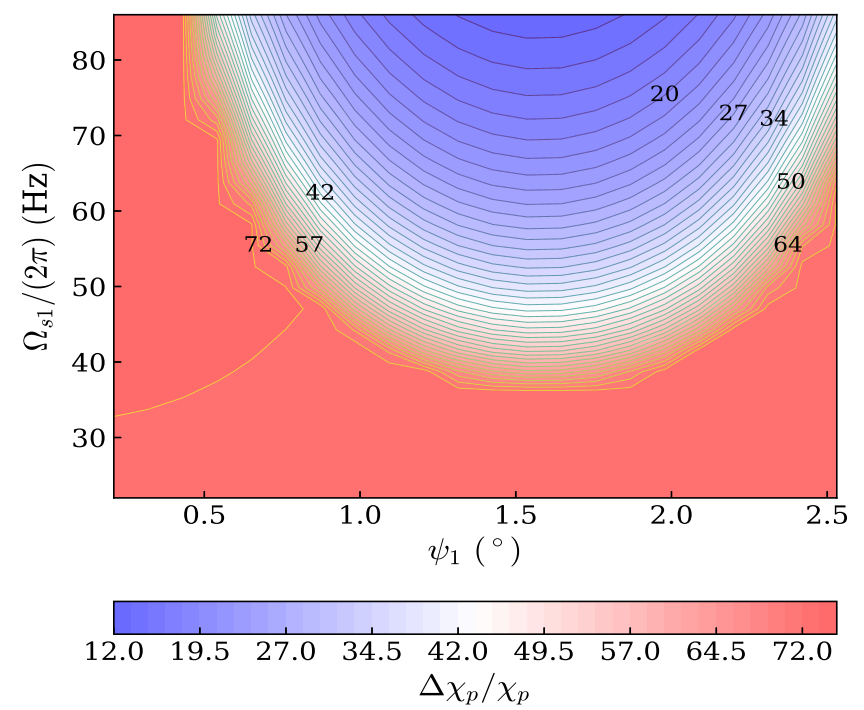

FIG. 20. The fractional error in inferring the PP precession parameter $\chi_{p}$.

For a typical NS with spin $<100 \mathrm{~Hz}$, it corresponds to a precession parameter $\chi_{\mathrm{p}} \lesssim 0.1$ (even assuming $\psi=\pi / 2 ; \chi_{\mathrm{p}}$ is smaller for harder EOSs). Consequently, neglecting precessions will cause only small errors on the PP waveforms.

Last, we show in Fig. 20 the parameter estimation uncertainties of $\chi_{\mathrm{p}}$ using the PP waveform alone. Here we have assumed a fiducial relation of $\chi_{\mathrm{p}}=0.06 \Omega_{s_{1}} \sin \psi_{1} /$ $(2 \pi \times 100 \mathrm{~Hz})$ so that we can show the $y$ axis in physical spin units. As the fractional error $\Delta \chi_{\mathrm{p}} / \chi_{\mathrm{p}}>10$ for the parameter space of interest, we do not expect we would be able to further improve the parameter estimation accuracy by incorporating the recession effects.

\section{APPENDIX B: THE TOLMAN-OPPENHEIMER- VOLKOFF EQUATIONS}

The stress-energy tensor $T_{\mu \nu}$ for a perfect fluid is given by

$$
T_{\mu \nu}=(\rho+p) u_{\mu} u_{\nu}+p g_{\mu \nu},
$$

where $p$ and $\rho$ stand for the pressure and energy density of the star and $u_{\mu}$ is the four-velocity. The metric $g_{\mu \nu}$ is given by Eq. (49). With the Einstein field equation, the quantities shown above can be solved by the TOV equations

$$
\begin{gathered}
\frac{d m}{d r}=4 \pi r^{2} \rho, \\
\frac{d p}{d r}=-\frac{\left(4 \pi r^{3} p+m\right)(\rho+p)}{r(r-2 m)}, \\
\frac{d \nu}{d r}=2 \frac{4 \pi r^{3} p+m}{r(r-2 m)},
\end{gathered}
$$

where 


$$
m \equiv \frac{1-e^{-\lambda}}{2} r .
$$

In practice, it is preferable to cast them into a new form for numerical integration. Following the procedure of Ref. [125], we use the specific enthalpy $h$, defined by

$$
d h=\frac{d p}{\rho+p},
$$

to replace $r$, where the integration constant is set by the condition $h \rightarrow 0$ as $\rho \rightarrow 0$ and $p \rightarrow 0$. Defining two new dependent variables, $u=r^{2}$ and $v=m / r$, we then have

$$
\begin{gathered}
\frac{d u}{d h}=-\frac{2 u(1-2 v)}{4 \pi u p+v}, \\
\frac{d v}{d h}=-(1-2 v) \frac{4 \pi u \rho-v}{4 \pi u p+v}, \\
\frac{d \nu}{d h}=-2 .
\end{gathered}
$$

At the center of the star, we have

$$
h=h_{c}, \quad u=0, \quad v=0,
$$

with $h_{c}$ a free parameter. The surface of the star is located at $h=0$.

Therefore, we can find the structure of the star by integrating Eqs. (B7)-(B9) from $h=h_{c}$ to $h=0$ with the initial conditions given in Eq. (B10). The total mass of the star can be obtained from the formula $m_{\mathrm{NS}}=\left.\sqrt{u} v\right|_{h=0}$, and the radius is given by $R_{\mathrm{NS}}=\left.\sqrt{u}\right|_{h=0}$. The quantity $\nu$ is linear in $h$, where the integration constant is set by the condition $\left.\nu\right|_{h=0}=\log \left(1-2 m_{\mathrm{NS}} / R_{\mathrm{NS}}\right)$ to connect the value outside of the star.

\section{APPENDIX C: THE CALCULATION OF TIDAL LOVE NUMBER $\lambda_{f}$}

Let us consider linearized even-parity perturbations to the equilibrium metric in Eq. (49). Following Refs. [126-128], we can write the perturbed metric in the Regge-Wheeler gauge as

$$
g_{\mu \nu}=g_{\mu \nu}^{(0)}+h_{\mu \nu}
$$

with

$$
h_{\mu \nu}=\operatorname{diag}\left[e^{-\nu} H_{0}, e^{\lambda} H_{2}, r^{2} K, r^{2} \sin ^{2} \theta K\right] Y_{l m}(\theta, \phi),
$$

where $\mathrm{H}_{0}, \mathrm{H}_{2}, \mathrm{~K}$ are functions of $r$. The perturbation on the stress-energy tensor is given by [126]

$$
\begin{gathered}
\delta T_{0}^{0}=-\delta \rho_{l} Y_{l m}(\theta, \phi)=-\frac{d \rho}{d p} \delta p_{l} Y_{l m}(\theta, \phi), \\
\delta T_{i}^{i}=\delta p_{l} Y_{l m}(\theta, \phi) .
\end{gathered}
$$

With the Einstein field equation, we obtain [128]

$$
\begin{aligned}
& H_{2}=H_{0}=H, \\
& H^{\prime \prime}+H^{\prime}\left\{\frac{2}{r}+e^{\lambda}\left[\frac{2 m}{r^{2}}+4 \pi r(p-\rho)\right]\right\} \\
& +H\left\{e^{\lambda}\left[-\frac{6}{r^{2}}+4 \pi(\rho+p) \frac{d \rho}{d p}+4 \pi(5 \rho+9 p)\right]\right. \\
& \left.-\left(\frac{d \nu}{d r}\right)^{2}\right\}=0,
\end{aligned}
$$

where we focus on the $l=2$ component. The mass function $m$ is related to the metric function $\lambda$ by Eq. (B5).

Imposing the regularity condition at $r=0$ yields the initial condition $H \propto r^{2}$. The proportionality constant does not matter here, so we simply set it at 1 . Functions $\lambda, m, p, \rho$ in the above equation can be obtained from the solutions of the TOV equations.

Integrating Eq. (C6) from $r=0$ to $r=R_{\mathrm{NS}}$ leads to the dimensionless tidal Love number $k_{2}$ as [129]

$$
\begin{aligned}
k_{2}= & \frac{8}{5} \mathcal{C}^{5}(1-2 \mathcal{C})^{2}[2+2 \mathcal{C}(y-1)-y]\{2 \mathcal{C}[6-3 y \\
& +3 \mathcal{C}(5 y-8)]+4 \mathcal{C}^{3}[13-11 y+\mathcal{C}(3 y-2) \\
& \left.+2 \mathcal{C}^{2}(1+y)\right]+3(1-2 \mathcal{C})^{2}[2-y+2 \mathcal{C}(y-1)] \\
& \times \log (1-2 \mathcal{C})\}^{-1},
\end{aligned}
$$

where $y=R_{\mathrm{NS}} H^{\prime}\left(R_{\mathrm{NS}}\right) / H\left(R_{\mathrm{NS}}\right)$ and the tidal Love number $\lambda_{f}$ is given by

$$
\lambda_{f}=\frac{2}{3} k_{2} R_{\mathrm{NS}}^{5} .
$$

\section{APPENDIX D: THE GW PHASE WITH SPA}

From Refs. [99-106], we obtain the frequency-domain gravitational-wave phasing, up to $3.5 \mathrm{PN}$ order for pointparticle contributions, up to $3 \mathrm{PN}$ for spin terms, and up to 2.5PN for an adiabatic, $f$-mode tide. Here are the terms in addition to the leading Newtonian phasing $\Psi_{N}$ :

$$
\begin{aligned}
\Psi_{\mathrm{PP}}= & \frac{3}{128}(\pi \mathcal{M} f)^{-5 / 3}\left\{\left(\frac{3715}{756}+\frac{55}{9} \eta\right) x-16 \pi x^{3 / 2}+\left(\frac{15293365}{508032}+\frac{27145}{504} \eta+\frac{3085}{72} \eta^{2}\right) x^{2}+\left(\frac{38645}{756} \pi-\frac{65}{9} \pi \eta\right)\right. \\
& \times(1+3 \log v) x^{5 / 2}+\left[\frac{11583231236531}{4694215680}-\frac{640 \pi^{2}}{3}-\frac{6848}{21} \gamma_{E}-\left(\frac{15737765635}{3048192}-\frac{2255}{12} \pi^{2}\right) \eta+\frac{76055}{1728} \eta^{2}-\frac{127825}{1296} \eta^{3}\right. \\
& \left.\left.-\frac{6848}{21} \log (4 v)\right] x^{3}+\left(\frac{77096675}{254016}+\frac{1014115}{3024} \eta-\frac{36865}{378} \eta^{2}\right) \pi x^{7 / 2}\right\}
\end{aligned}
$$




$$
\begin{aligned}
\Psi_{S O}= & \frac{3}{128}(\pi \mathcal{M} f)^{-5 / 3}\left\{4\left(\frac{113}{12}-\frac{19}{3} \eta\right)\left(\hat{\boldsymbol{L}} \cdot \chi_{s}\right) x^{3 / 2}-10\left[\frac{719}{48} \delta_{m}\left(\hat{\boldsymbol{L}} \cdot \chi_{s}\right)\left(\hat{\boldsymbol{L}} \cdot \chi_{a}\right)+\left(\frac{719}{96}+\frac{\eta}{24}\right)\left(\hat{\boldsymbol{L}} \cdot \chi_{s}\right)^{2}+\left(\frac{719}{96}-30 \eta\right)\right.\right. \\
& \left.\times\left(\hat{\boldsymbol{L}} \cdot \chi_{a}\right)^{2}\right] x^{2}-(1+3 \log v)\left[\left(\frac{732985}{2268}-\frac{24260}{81} \eta-\frac{340}{9} \eta^{2}\right)\left(\hat{\boldsymbol{L}} \cdot \chi_{s}\right)+\left(\frac{732985}{2268}+\frac{140}{9} \eta\right) \delta_{m}\left(\hat{\boldsymbol{L}} \cdot \chi_{a}\right)\right] x^{5 / 2} \\
& \left.+\frac{2270 \pi}{3}\left[\left(1-\frac{227}{156} \eta\right)\left(\hat{\boldsymbol{L}} \cdot \chi_{s}\right)+\delta_{m}\left(\hat{\boldsymbol{L}} \cdot \chi_{a}\right)\right] x^{3}\right\}, \\
\Psi_{\bar{\lambda}_{f}^{(1)}}= & -\frac{3}{16 \eta} x^{5 / 2}(12-11 \Xi) \bar{\lambda}_{f}^{(1)} \Xi^{4}\left\{1+\frac{5\left(3179-919 \Xi-2286 \Xi^{2}+260 \Xi^{3}\right)}{672(12-11 \Xi)} x-\pi x^{3 / 2}\right. \\
& +\frac{1}{12-11 \Xi}\left[\frac{39927845}{508032}-\frac{480043345}{9144576} \Xi+\frac{9860575}{127008} \Xi^{2}-\frac{421821905}{2286144} \Xi^{3}+\frac{4359700}{35721} \Xi^{4}-\frac{10578445}{285768} \Xi^{5}\right] x^{2} \\
& \left.-\frac{\pi\left(27719-22127 \Xi+7022 \Xi^{2}-10232 \Xi^{3}\right)}{672(12-11 \Xi)} x^{5 / 2}\right\},
\end{aligned}
$$

$$
\Psi_{\bar{\lambda}_{f}^{(2)}}=(1 \rightarrow 2 \quad \text { and } \quad \Xi \rightarrow 1-\Xi) . \quad(\mathrm{D} 1 \mathrm{c})
$$

Here we have defined $x=v^{2}=(\pi M f)^{2 / 3}, \Xi=m_{1} / M$, $\delta_{m}=\left(m_{1}-m_{2}\right) / M . \hat{L}$ is the unit vector along the orbital angular momentum. Symmetric and antisymmetric dimensionless spins are defined as $\chi_{s}=\left(\chi_{1}+\chi_{2}\right) / 2$ and $\chi_{a}=$ $\left(\chi_{1}-\chi_{2}\right) / 2$ with $\chi_{i}=\bar{I}_{i} \boldsymbol{\Omega}_{s i} m_{i}$. Here we use the normalized momentum of inertia, as well as the normalized tidal Love number in Eqs. (D1b) and (D1c), in order to employ the I-Love universal relation.

\section{APPENDIX E: CASE I FOR FPS EOS (WITHOUT UNIVERSAL RELATIONS)}

In Fig. 21, we present the results for a BNS system with the FPS EOS. Following Sec. VII B, we still assume that both NSs are excited before the merger. The spin vector of $m_{2}$ is fixed at $\Omega_{s 2}=2 \pi \times 40 \mathrm{~Hz}$ and $\psi_{2}=7 \pi / 18$, and we vary the spin of $m_{1}: \Omega_{s 1} \in 2 \pi \times[10,85] \mathrm{Hz}$, $\psi_{1} \in\left[\frac{1}{20} \pi, \frac{17}{20} \pi\right]$. Without universal relations, we can put constraints on $\Omega_{s i}$ and $\mathcal{I}_{i}$. From Table VI, we know that the constraints for FPS are worse than those for GM1 by a factor of $\sim 2.6-3.2$. But their dependence on $\Omega_{s 1}$ and $\psi_{s 1}$ is the same as in the case of GM1.
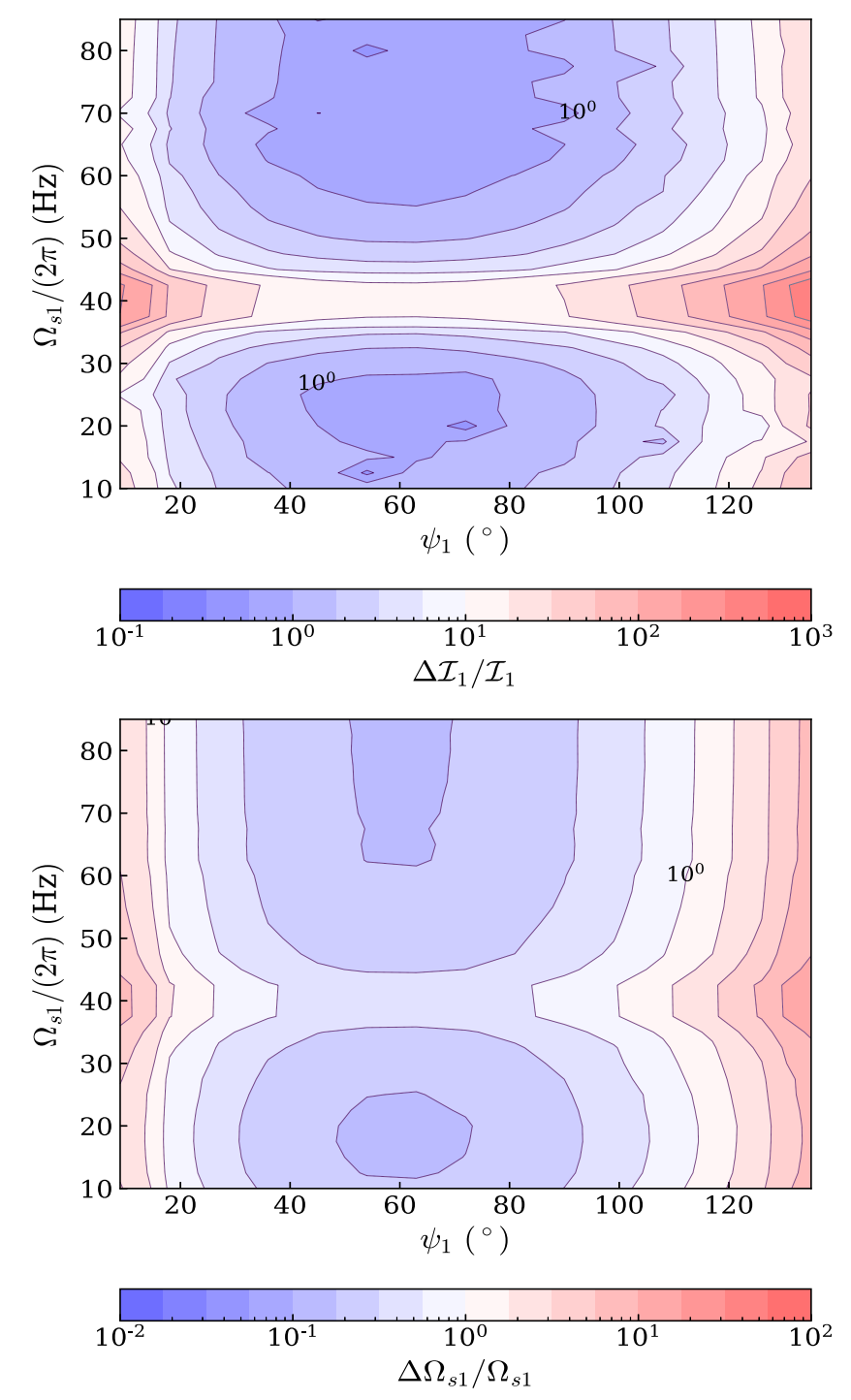

FIG. 21. Same as Fig. 11, but with the FPS EOS. The constraints are worse than those for GM1 by a factor of 2.6-2.7. 


\section{APPENDIX F: CASE II FOR FPS EOS (WITH UNIVERSAL RELATIONS)}

After incorporating universal relations into the calculations of Appendix E, we can obtain the constraints on $\bar{\lambda}_{f}^{s(a)}, \Xi$, and $\psi_{i}$, as shown in Figs. 22 and 23.
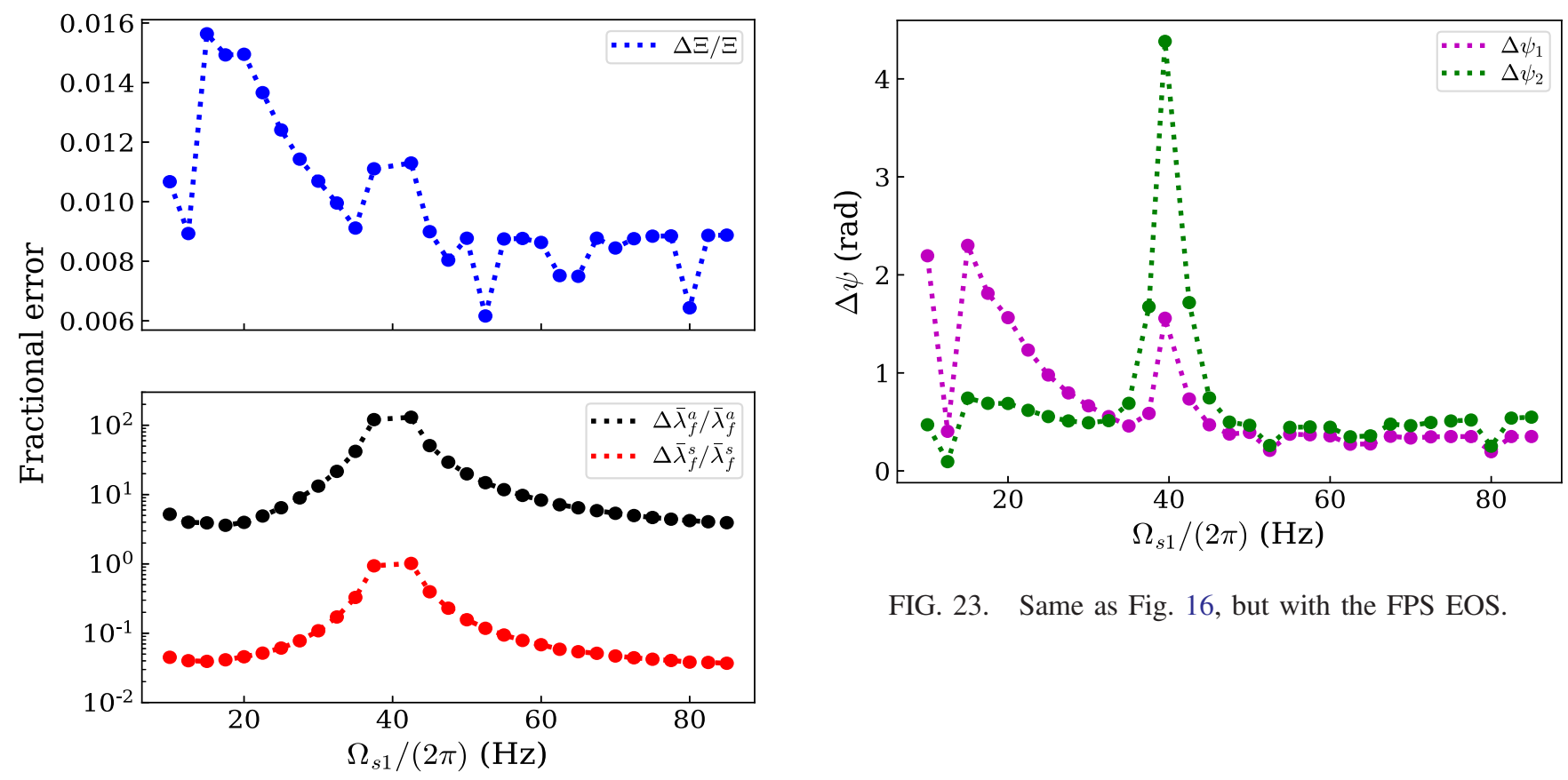

FIG. 23. Same as Fig. 16, but with the FPS EOS.

FIG. 22. Same as Fig. 13, but with the FPS EOS.

[1] B. P. Abbott et al. (LIGO Scientific and Virgo Collaborations), Phys. Rev. Lett. 119, 161101 (2017).

[2] B. P. Abbott et al. (LIGO Scientific, Virgo, Fermi-GBM, and INTEGRAL Collaborations), Astrophys. J. 848, L13 (2017).

[3] B. P. Abbott et al. (LIGO Scientific and Virgo Collaborations), Astrophys. J. Lett. 892, L3 (2020).

[4] B. P. Abbott et al. (LIGO Scientific and Virgo Collaborations), Phys. Rev. Lett. 121, 161101 (2018).

[5] B. P. Abbott et al. (LIGO Scientific and Virgo Collaborations), Classical Quant. Grav. 37, 045006 (2020).

[6] E. Annala, T. Gorda, A. Kurkela, and A. Vuorinen, Phys. Rev. Lett. 120, 172703 (2018).

[7] E. R. Most, L. R. Weih, L. Rezzolla, and J. SchaffnerBielich, Phys. Rev. Lett. 120, 261103 (2018).

[8] B. Margalit and B. D. Metzger, Astrophys. J. 850, L19 (2017).

[9] L. Rezzolla, E. R. Most, and L. R. Weih, Astrophys. J. 852, L25 (2018).

[10] M. Ruiz, S. L. Shapiro, and A. Tsokaros, Phys. Rev. D 97, 021501 (2018).
[11] M. Shibata, S. Fujibayashi, K. Hotokezaka, K. Kiuchi, K. Kyutoku, Y. Sekiguchi, and M. Tanaka, Phys. Rev. D 96, 123012 (2017).

[12] G. Pratten, P. Schmidt, and T. Hinderer, Nat. Commun. 11, 2553 (2020)

[13] J. Aasi et al. (LIGO Scientific Collaboration), Classical Quant. Grav. 32, 115012 (2015).

[14] M. Tse et al., Phys. Rev. Lett. 123, 231107 (2019).

[15] G. M. Harry (LIGO Scientific Collaboration), Classical Quant. Grav. 27, 084006 (2010).

[16] B. P. Abbott et al. (KAGRA, LIGO Scientific, and Virgo Collaborations), Living Rev. Relativity 21, 3 (2018).

[17] See https://dcc.ligo.org/ligo-m060056/public.

[18] See https://dcc.ligo.org/ligo-t1800044/public.

[19] D. Shoemaker (LIGO Scientific Collaboration), arXiv: 1904.03187.

[20] See https://dcc.ligo.org/ligo-t1600119/public.

[21] See https://tds.virgo-gw.eu/?call_file=vir-0027a-09.pdf.

[22] F. Acernese et al. (Virgo Collaboration), Classical Quant. Grav. 32, 024001 (2015). 
[23] F. Acernese et al. (Virgo Collaboration), Phys. Rev. Lett. 123, 231108 (2019).

[24] See https://tds.ego-gw.it/ql/?c=8940.

[25] See https://dcc.ligo.org/ligo-m1100296/public.

[26] Y. Aso, Y. Michimura, K. Somiya, M. Ando, O. Miyakawa, T. Sekiguchi, D. Tatsumi, and H. Yamamoto (KAGRA Collaboration), Phys. Rev. D 88, 043007 (2013).

[27] K. Somiya (KAGRA Collaboration), Classical Quant. Grav. 29, 124007 (2012).

[28] M. Punturo et al., Classical Quant. Grav. 27, 084007 (2010).

[29] S. Hild et al., Classical Quant. Grav. 28, 094013 (2011).

[30] See http://www.et-gw.eu/index.php/etdsdocument.

[31] S. Hild, S. Chelkowski, A. Freise, J. Franc, N. Morgado, R. Flaminio, and R. DeSalvo, Classical Quant. Grav. 27, 015003 (2010).

[32] B. P. Abbott et al. (LIGO Scientific Collaboration), Classical Quant. Grav. 34, 044001 (2017).

[33] D. Reitze et al., Bull. Am. Astron. Soc. 51, 035 (2019).

[34] V. Baibhav, E. Berti, D. Gerosa, M. Mapelli, N. Giacobbo, Y. Bouffanais, and U. N. Di Carlo, Phys. Rev. D 100, 064060 (2019).

[35] T. Hinderer et al., Phys. Rev. Lett. 116, 181101 (2016).

[36] T. Dietrich, S. Bernuzzi, and W. Tichy, Phys. Rev. D 96, 121501 (2017).

[37] S. Akcay, S. Bernuzzi, F. Messina, A. Nagar, N. Ortiz, and P. Rettegno, Phys. Rev. D 99, 044051 (2019).

[38] A. Nagar et al., Phys. Rev. D 98, 104052 (2018).

[39] K. Barkett, Y. Chen, M. A. Scheel, and V. Varma, Phys. Rev. D 102, 024031 (2020).

[40] F. Messina, R. Dudi, A. Nagar, and S. Bernuzzi, Phys. Rev. D 99, 124051 (2019).

[41] T. G. Cowling, Mon. Not. R. Astron Soc. 101, 367 (1941).

[42] A. Reisenegger and P. Goldreich, Astrophys. J. 426, 688 (1994).

[43] D. Lai, Mon. Not. R. Astron. Soc. 270, 611 (1994).

[44] H. Yu and N. N. Weinberg, Mon. Not. R. Astron Soc. 464, 2622 (2017).

[45] H. Yu and N. N. Weinberg, Mon. Not. R. Astron. Soc. 470, 350 (2017).

[46] C. Chirenti, R. Gold, and M. Miller, Astrophys. J. 837, 67 (2017).

[47] H. Yang, W. E. East, V. Paschalidis, F. Pretorius, and R. F. P. Mendes, Phys. Rev. D 98, 044007 (2018).

[48] H. Yang, Phys. Rev. D 100, 064023 (2019).

[49] M. Vick and D. Lai, Phys. Rev. D 100, 063001 (2019).

[50] D. Lai, Astrophys. J. 466, L35 (1996).

[51] D. Lai, Astrophys. J. 490, 847 (1997).

[52] W. C. G. Ho and D. Lai, Mon. Not. R. Astron. Soc. 308, 153 (1999).

[53] S. Ma, H. Yu, and Y. Chen, Phys. Rev. D 101, 123020 (2020).

[54] L. Gualtieri, E. Berti, J. A. Pons, G. Miniutti, and V. Ferrari, Phys. Rev. D 64, 104007 (2001).

[55] J. A. Pons, E. Berti, L. Gualtieri, G. Miniutti, and V. Ferrari, Phys. Rev. D 65, 104021 (2002).

[56] G. Miniutti, J.A. Pons, E. Berti, L. Gualtieri, and V. Ferrari, Mon. Not. R. Astron. Soc. 338, 389 (2003).

[57] J. Steinhoff, T. Hinderer, A. Buonanno, and A. Taracchini, Phys. Rev. D 94, 104028 (2016).
[58] P. Schmidt and T. Hinderer, Phys. Rev. D 100, 021501 (2019).

[59] A. G. Lyne et al., Science 303, 1153 (2004).

[60] N. Andersson and W. C. G. Ho, Phys. Rev. D 97, 023016 (2018).

[61] L. Lindblom and J. R. Ipser, Phys. Rev. D 59, 044009 (1999).

[62] K. H. Lockitch and J. L. Friedman, Astrophys. J. 521, 764 (1999).

[63] S. Yoshida and U. Lee, Astrophys. J. 529, 997 (2000).

[64] J. Papaloizou and J. Pringle, Mon. Not. R. Astron. Soc. 182, 423 (1978).

[65] H. Saio, Astrophys. J. 256, 717 (1982).

[66] K. H. Lockitch, N. Andersson, and J. L. Friedman, Phys. Rev. D 63, 024019 (2000).

[67] K. H. Lockitch, J. L. Friedman, and N. Andersson, Phys. Rev. D 68, 124010 (2003).

[68] A. Idrisy, B. J. Owen, and D. I. Jones, Phys. Rev. D 91, 024001 (2015).

[69] D. Lai and Y. Wu, Phys. Rev. D 74, 024007 (2006).

[70] W. Xu and D. Lai, Phys. Rev. D 96, 083005 (2017).

[71] A. K. Schenk, P. Arras, E. E. Flanagan, S. A. Teukolsky, and I. Wasserman, Phys. Rev. D 65, 024001 (2001).

[72] E. E. Flanagan and E. Racine, Phys. Rev. D 75, 044001 (2007).

[73] E. Poisson, Phys. Rev. D 101, 104028 (2020).

[74] E. Poisson, Phys. Rev. D 102, 064059 (2020).

[75] E. Poisson and C. Buisson, Phys. Rev. D 102, 104005 (2020).

[76] H. Yu et al., Phys. Rev. Lett. 120, 141102 (2018).

[77] K. Yagi and N. Yunes, Phys. Rev. D 88, 023009 (2013).

[78] K. Yagi and N. Yunes, Science 341, 365 (2013).

[79] A. Nagar, F. Messina, P. Rettegno, D. Bini, T. Damour, A. Geralico, S. Akcay, and S. Bernuzzi, Phys. Rev. D 99, 044007 (2019).

[80] P. Schmidt, F. Ohme, and M. Hannam, Phys. Rev. D 91, 024043 (2015).

[81] E. E. Flanagan and T. Hinderer, Phys. Rev. D 77, 021502 (2008).

[82] E. Racine, Phys. Rev. D 78, 044021 (2008).

[83] E. Racine and E. E. Flanagan, Phys. Rev. D 71, 044010 (2005); 88, 089903(E) (2013).

[84] J. Provost, G. Berthomieu, and A. Rocca, Astron. Astrophys. 94, 126 (1981).

[85] E. Poisson and C. M. Will, Gravity: Newtonian, PostNewtonian, Relativistic (Cambridge University Press, Cambridge, England, 2014).

[86] S. Chandrasekhar, Phys. Rev. Lett. 24, 611 (1970).

[87] J. L. Friedman and B. F. Schutz, Astrophys. J. 222, 281 (1978).

[88] R. Boyer and R. Lindquist, Phys. Lett. 20, 504 (1966).

[89] J. B. Hartle and D. H. Sharp, Astrophys. J. 147, 317 (1967).

[90] A. Akmal, V. R. Pandharipande, and D. G. Ravenhall, Phys. Rev. C 58, 1804 (1998).

[91] V. Pandharipande and D. Ravenhall, in Nuclear Matter and Heavy Ion Collisions (Springer, New York, 1989), pp. $103-132$.

[92] N. K. Glendenning and S. A. Moszkowski, Phys. Rev. Lett. 67, 2414 (1991). 
[93] F. Douchin and P. Haensel, Astron. Astrophys. 380, 151 (2001).

[94] H. Togashi, K. Nakazato, Y. Takehara, S. Yamamuro, H. Suzuki, and M. Takano, Nucl. Phys. A961, 78 (2017).

[95] G. Baym, T. Hatsuda, T. Kojo, P. D. Powell, Y. Song, and T. Takatsuka, Rep. Prog. Phys. 81, 056902 (2018).

[96] G. Baym, S. Furusawa, T. Hatsuda, T. Kojo, and H. Togashi, Astrophys. J. 885, 42 (2019).

[97] P. Haensel and A. Y. Potekhin, Astron. Astrophys. 428, 191 (2004).

[98] See https://compose.obspm.fr/home/.

[99] K. G. Arun, B. R. Iyer, B. S. Sathyaprakash, and P. A. Sundararajan, Phys. Rev. D 71, 084008 (2005); 72, 069903(E) (2005).

[100] L. E. Kidder, C. M. Will, and A. G. Wiseman, Phys. Rev. D 47, R4183 (1993).

[101] K. G. Arun, A. Buonanno, G. Faye, and E. Ochsner, Phys. Rev. D 79, 104023 (2009); 84, 049901(E) (2011).

[102] L. Blanchet, A. Buonanno, and G. Faye, Phys. Rev. D 84, 064041 (2011).

[103] B. Mikóczi, M. Vasúth, and L. Á. Gergely, Phys. Rev. D 71, 124043 (2005).

[104] T. Hinderer, Astrophys. J. 677, 1216 (2008).

[105] T. Damour, A. Nagar, and L. Villain, Phys. Rev. D 85, 123007 (2012).

[106] Q. Henry, G. Faye, and L. Blanchet, Phys. Rev. D 102, 044033 (2020).

[107] B. P. Abbott et al. (LIGO Scientific Collaboration), Classical Quant. Grav. 34, 044001 (2017).

[108] I. S. Shklovskii, Sov. Astron. 13, 562 (1970).

[109] N. Brandt and P. Podsiadlowski, Mon. Not. R. Astron Soc. 274, 461 (1995).

[110] R. G. Martin, C. A. Tout, and J. E. Pringle, Mon. Not. R. Astron Soc. 397, 1563 (2009).
[111] H.-T. Janka, K. Langanke, A. Marek, G. Martinez-Pinedo, and B. Mueller, Phys. Rep. 442, 38 (2007).

[112] A. Burrows, Rev. Mod. Phys. 85, 245 (2013).

[113] F. Foucart et al., Phys. Rev. D 99, 044008 (2019).

[114] See https://www.black-holes.org/code/spec.html.

[115] L. E. Kidder et al., J. Comput. Phys. 335, 84 (2017).

[116] D. G. Yakovlev, K. P. Levenfish, and Y. A. Shibanov, Phys. Usp. 42, 737 (1999).

[117] N. Andersson and G. L. Comer, Mon. Not. R. Astron Soc. 328, 1129 (2001).

[118] L. Lindblom and G. Mendell, Phys. Rev. D 61, 104003 (2000).

[119] N. Andersson and K. D. Kokkotas, Int. J. Mod. Phys. D 10, 381 (2001).

[120] Z. Carson, K. Chatziioannou, C.-J. Haster, K. Yagi, and N. Yunes, Phys. Rev. D 99, 083016 (2019).

[121] B. M. Barker and R. F. O'Connell, Phys. Rev. D 12, 329 (1975).

[122] H. Yu, S. Ma, M. Giesler, and Y. Chen, Phys. Rev. D 102, 123009 (2020).

[123] LIGO Scientific Collaboration, LIGO Algorithm Library, LALSuite (GPL), 2018.

[124] M. Hannam, P. Schmidt, A. Bohé, L. Haegel, S. Husa, F. Ohme, G. Pratten, and M. Pürrer, Phys. Rev. Lett. 113, 151101 (2014).

[125] L. Lindblom, Phys. Rev. D 58, 024008 (1998).

[126] K. S. Thorne and A. Campolattaro, Astrophys. J. 149, 591 (1967).

[127] J. R. Ipser and R. H. Price, Phys. Rev. D 43, 1768 (1991).

[128] L. Lindblom, G. Mendell, and J. R. Ipser, Phys. Rev. D 56, 2118 (1997).

[129] T. Hinderer, Astrophys. J. 677, 1216 (2008). 